\title{
RGD-Functionalized Melanin Nanoparticles for Intraoperative Photoacoustic Imaging-Guided Breast Cancer Surgery
}

Jing-Jing Liu

Xiamen University

\section{Zun Wang}

Shantou University Medical College

Li-Ming Nie

Xiamen University

Yuan-Yuan Zhu

Xiamen University

Ge Li

Xiamen University

Lin-Ling Lin

Xiamen University

Min Chen

Xiamen University

Guo-Jun Zhang ( $\sim$ guoj_zhang@yahoo.com )

Xiamen University https://orcid.org/0000-0001-5182-5887

\section{Research Article}

Keywords: melanin, bioimaging, photoacoustic imaging, breast cancer, tumour 59 detection, imagingguided surgery

Posted Date: April 26th, 2021

DOI: https://doi.org/10.21203/rs.3.rs-429256/v1

License: (c) (i) This work is licensed under a Creative Commons Attribution 4.0 International License. Read Full License 


\section{RGD-functionalized melanin nanoparticles for intraoperative}

photoacoustic imaging-guided breast cancer surgery

Jing-Jing Liu' ${ }^{1,2, \#}$, Zun Wang ${ }^{5,7 \#}$, Li-Ming Nie ${ }^{6,8}$, Yuan-Yuan $\mathrm{Zhu}^{1,2}, \mathrm{Ge} \mathrm{Li}^{1,2}$, Lin-Ling Lin ${ }^{1,2}$, Min Chen ${ }^{2,3,4,{ }^{*}}$ and Guo-Jun Zhang ${ }^{1,2,3, *}$

${ }^{1}$ Cancer Center \& Department of Breast and Thyroid Surgery, Xiang'an Hospital of Xiamen University, School of Medicine, Xiamen University, 2000 Xiang'an Road East, Xiamen, 361101, Fujian, China.

${ }^{2}$ Key Laboratory for Endocrine-Related Cancer Precision Medicine of Xiamen, Xiang'an Hospital of Xiamen University, Xiamen, Fujian, China.

${ }^{3}$ Cancer Research Center, School of Medicine, Xiamen University, Xiamen, China.

${ }^{4}$ Clinical Central Research Core, Xiang'an Hospital of Xiamen University, No. 2000, Xiang'an Road East, Xiamen, 361101, Fujian, China.

${ }^{5}$ ChangJiang Scholar's Laboratory, Shantou University Medical College, 515041, Shantou, China.

${ }^{6}$ State Key Laboratory of Molecular Vaccinology and Molecular Diagnosis \& Center for Molecular Imaging and Translational Medicine, School of Public Health, Xiamen University, Xiamen, 361102, China.

\# These authors contributed equally to this work.

* Corresponding authors: Guojun Zhang, Phone: 0086-592-28899988, e-mail: gjzhang@xah.xmu.edu.cn; Min Chen, Phone: 0086-0592-2889270, e-mail: mchen@xah.xmu.edu.cn;

Note: Present Address:

${ }^{7}$ Department of Breast and Thyroid Surgery, Shenzhen Baoan Women's and Children's Hospital, Jinan University, 518133, Shenzhen, China.

8 Department of Radiology and Optical Imaging Laboratory, Guangdong Provincial People's Hospital, Guangdong Academy of Medical Sciences, 510080, Guangzhou, China. 


\begin{abstract}
Purpose: Obtaining tumour-free margins is critical for avoiding re-excision and reducing local recurrence following breast-conserving surgery (BCS); however, it remains challenging. Imaging-guided surgery provides precise detection of residual lesions and assists surgical resection. Herein, we describe water-soluble melanin nanoparticles (MNPs) conjugated with cyclic Arg-Gly-Asp (cRGD) peptides for breast cancer photoacoustic imaging (PAI) and surgery navigation.
\end{abstract}

Methods: cRGD-MNPs was synthesized and characterized for morphology, photoacoustic characteristics and stability. Tumour targeting and toxicity were determined by cells and tumour-bearing mice. PAI was used to locate the tumour and guide surgical resection in MDA-MB-231 tumour-bearing mice.

Results: The cRGD-MNPs exhibited excellent tumour-targeting in vitro and in vivo, with low toxicity. Intravenous administration of cRGD-MNPs to MDA-MB-231 tumour-bearing mice showed an approximately 2.1-fold enhancement in photoacoustic (PA) intensity at $2 \mathrm{~h}$, and the ratio of the PA intensity at the tumour site compared to that in the surrounding normal tissue was $3.2 \pm 0.1$, which was much higher than that using MNPs alone $(1.7 \pm 0.3)$. Similarly, the PA signal in the mammary glands containing spontaneous breast cancer was enhanced (2.5 \pm 0.3 -fold) in MMTV-PyVT transgenic murine model. Preoperative screening by PAI could assess tumour volume and offer a three-dimensional (3D) reconstruction image for accurate surgical planning. Surgical resection following real-time PAI on the tumour bed showed high consistency with histopathological analysis.

Conclusion: These results highlight that cRGD-MNPs combined with PAI provide a powerful tool for breast cancer imaging and precise tumour resection. cRGD-MNPs with good PA properties have great potential for clinical translation.

Keywords: melanin, bioimaging, photoacoustic imaging, breast cancer, tumour detection, imaging-guided surgery 


\section{Introduction}

Nearly half of patients with early breast cancer undergo breast-conserving surgery (BCS) with adjuvant radiation therapy [1]. BCS involves the removal of the primary tumour with a margin of surrounding normal breast tissue. Although BCS is the standard of care treatment for early breast cancer patients [2], a potential downside is the failure to achieve clear or negative margins with lumpectomy. In particular, 20-40\% of patients require additional operative intervention by re-excision or even mastectomy [3]. Re-excision surgery increases the risk of complications (e.g., infection and unsatisfactory aesthetic results) and potentially postpones systemic treatment. It also increases health care costs and burden and harms patients both physically and psychologically.

During BCS, surgeons typically use visual inspection and tactile feedback to determine the tumour location and set resection margins. However, it is difficult to discriminate tumour margins from surrounding normal tissue macroscopically. Frozen section and imprint cytology are applied clinically for intraoperative margin assessment. These methods have the potential to lower the rates of positive margins, but they are labour-intensive and time-consuming and have low sensitivity due to sampling rate limitations $[4,5]$. Currently, there have been multiple emerging intraoperative imaging tools for real-time margin assessment, including wire-guided localization [6], intraoperative ultrasound [7] and postoperative techniques (intraoperative specimen mammography/micro-computed tomography) [8]. Due to their anatomical imaging modalities and limited tumour specificity, these methods have not been widely used for margin assessment. Fluorescence-guided surgical navigation is a high sensitivity technique using the injection of a fluorescent contrast agent that is a promising intraoperative tool for precise tumour resection [9]. However, this technique is hampered by a limited penetration depth because of light scattering and signal attenuation [10].

Recently, photoacoustic imaging (PAI) has been developed as a novel imaging technology for biomedical applications. PAI detects optical absorption contrast acoustically via the photoacoustic (PA) effect, a physical phenomenon that converts 
absorbed optical energy into acoustic energy [11]. Based on endogenous contrast molecules (e.g., oxyhaemoglobin, deoxyhaemoglobin, lipid, or DNA-RNA), PAI has been used in the clinic trial to demonstrate its highly desirable capabilities for breast cancer imaging in vivo and ex vivo, particularly for assessing tumour margins macroscopically and microscopically [12-14]. However, intrinsic chromophores provide access to only a limited range of biological processes but low tumour-imaging contrast. Hence, molecular PAI for breast cancer still requires a targeted contrast agent that can selectively bind to surface receptors on cancer cells or respond to the tumour microenvironment $[11,15]$.

Natural melanin is a group of biopigments with multifunctionality (i.e., ultraviolet protection, radical scavenging, and photothermal conversion) [16]. Due to its good intrinsic biocompatibility, natural melanin or synthetic melanin-like nanomaterials have been successfully developed as novel nano-bioplatforms in bioimaging, therapy, theranostics, and biosensing $[17,18]$. As an endogenous PA contrasting agent, melanin was used to detect the metastatic status of ex vivo human melanoma sentinel lymph nodes by multispectral optoacoustic imaging[19]. The results showed an excellent correlation with the histological assessment of melanoma cell infiltration with $100 \%$ sensitivity and $62 \%$ specificity [19].

In this study, we conducted a comprehensive study of the feasibility of the PAI method for improving the detection and accurate removal of breast cancer using targeted melanin nanoparticles (MNPs) conjugated with cyclic Arg-Gly-Asp (cRGD) (Scheme 1). Our data support the potential clinical application of cRGD-MNPs as a novel tumour-specific PA contrast agent for imaging-guided BCS. 


\section{Materials and methods}

\section{Reagents}

Melanin was obtained from Sigma-Aldrich. Sodium hydroxide, hydrochloric acid and $\mathrm{NH}_{4} \mathrm{OH}$ solution (28 wt\%) were purchased from Sinopharm Chemical Reagent Co., Ltd. Amine-PEG 5000 -amine $\left(\mathrm{NH}_{2}-\mathrm{PEG}_{5000}-\mathrm{NH}_{2}, 5 \mathrm{kDa}\right)$ was obtained from Shanghai Aladdin Bio-Chem Technology Co., LTD. CellTiter 96 AQueous One Solution Cell Proliferation Assay (MTS) was purchased from Promega, phosphate-buffered saline (PBS) was purchased from Solarbio and c(RGDfC) was from GL Biochem. Melanin staining kit was purchased from Servicebio. Dulbecco's modified eagle's medium (DMEM), fetal bovine serum (FBS), and penicillin-streptomycin were purchased from Gibco. Mammary Epithelial Cell Growth Medium (MEGM) BulletKit was purchased from Lonza.

\section{Cell culture}

The human breast cancer cell line MDA-MB-231 and non-cancerous mammary epithelial cell line MCF-10A were purchased from Procell Life Science \& Technology Co., Ltd. , and cells with passage number $<25$ were used in the experiment. MDA-MB231 cells were cultured in DMEM supplemented with 10\% FBS and 1\% penicillinstreptomycin. MCF-10A cells were cultured in MEGM supplemented with BPE, hEGF, insulin, hydrocortisone, and GA-1000. The cells were grown as sub-confluent monolayers at $37{ }^{\circ} \mathrm{C}$ under the water-saturated environment with $5 \% \mathrm{CO}_{2}$.

\section{Animals}

All animal experiments were performed in compliance with the Guidelines for the Care and Use of Research Animals established by the Xiamen University Animal Studies Committee. Female BALB/c nude mice and BALB/c mice (4-6 weeks, approximately $20 \mathrm{~g}$ ) were purchased from the Experimental Animal Center of Xiamen University and kept under sterile conditions. FVB/N-Tg(MMTV-PyVT)634Mul/J transgenic mice were purchased from the Jackson Laboratory and raised under specificpathogen-free conditions. 


\section{Preparation of cRGD-MNPs}

144

The cRGD-MNPs were obtained as previously described [20]. Briefly, melanin (2 $\mathrm{mg} / \mathrm{mL})$ was dissolved in $\mathrm{NaOH}$ aqueous solution $(0.1 \mathrm{~N})$ followed by the rapid addition of $\mathrm{HCl}$ aqueous solution $(0.1 \mathrm{~N})$ to a $\mathrm{pH}$ of 7.0 under sonication. The neutralised solution was centrifuged and washed with deionized water several times. The black solid of MNPs was obtained by freeze-drying. The MNPs aqueous solution $(1 \mathrm{mg} / \mathrm{mL}, \mathrm{pH}=9)$ was added dropwise into an $\mathrm{NH}_{2}-\mathrm{PEG}_{5000}-\mathrm{NH}_{2}$ aqueous solution. After vigorous stirring for $12 \mathrm{~h}$, the PEG-modified MNPs were retrieved by centrifugation and washed several times to remove the unreacted $\mathrm{NH}_{2}-\mathrm{PEG}_{5000}-\mathrm{NH}_{2}$. The PEGylated MNPs $(1 \mathrm{mg} / \mathrm{mL}, \mathrm{pH}=7.2)$ were incubated with 4-(Nmaleimidomethyl)cyclohexane-1-carboxylic acid 3-sulfo-N-hydroxysuccinimide ester sodium salt (sulfo-SMCC) solution (1.2 mg in $36 \mu \mathrm{L}$ of dimethyl sulfoxide) for $2 \mathrm{~h}$ at room temperature. The complexes were purified using a PD-10 column. The c(RGDfC) solution $(120 \mu \mathrm{L}$ of $5 \mathrm{mM}$ in degassed water) was added to the purified MNPs with stirring for $24 \mathrm{~h}$ at $4^{\circ} \mathrm{C}$. The excessive RGD peptide was removed using a PD-10 column. The final cRGD-MNPs were filtered through a $0.22 \mu \mathrm{m}$ filter.

\section{Characterisation of MNPs}

Transmission electron microscopy images were recorded using a Talos F200s transmission electron microscope (FEI, USA) at an accelerating voltage of $100 \mathrm{kV}$. The MNPs or cRGD-MNPs aqueous solution was dropped onto a carbon-coated copper grid and air-dried. The ${ }^{1} \mathrm{H}$ NMR spectra were recorded at $20^{\circ} \mathrm{C}$ on a $400 \mathrm{MHz} \mathrm{NMR}$ spectrometer (Bruker) using $\mathrm{D}_{2} \mathrm{O}$ as the solvent. Zeta potentials were measured using a laser particle size analyser system (Malvern, Zetasizer Nano ZS90). The absorption spectra were obtained using a Multiskan Spectrum Microplate Spectrophotometer (Thermo, USA).

\section{PAI system}

Both PA and US images were recorded using the Vevo LAZR-X photoacoustic imaging system (VisualSonics, FujiFilm, Japan). A tunable laser (680-970 nm) with a 
repetition rate of $20 \mathrm{~Hz}$ and a 256-element linear array with the main frequency of 40 $\mathrm{MHz}$ was used to obtain single-plane, full-spectrum and 3D PA images. The 3D scanning was controlled using an electric motor with a step length of $0.14 \mathrm{~mm}$. The quantified PA signal intensities within the region of interest (ROI) of each image were analysed using the Vevo LAB tool (VisualSonics, FujiFilm, Japan).

\section{PA signal of cRGD-MNPs}

For evaluating the PA performance of nanoparticles, MNPs and cRGD-MNPs (120 $\mu \mathrm{M})$ were scanned for PAI at different wavelengths ranging from 680 to $970 \mathrm{~nm}$ (interval $=5 \mathrm{~nm}$ ) to detect the optimum excitation wavelength. Different concentrations (3.75-120 $\mu \mathrm{M})$ of MNPs and cRGD-MNPs were dispersed in PBS and triggered by the optimal excitation wavelength to acquire the corresponding PA images. For photostability analysis, PA images of the MNPs and cRGD-MNPs solutions (120 $\mu \mathrm{M})$ were obtained at different time points (0, 2, 4, 6 and 7 days) using a $680 \mathrm{~nm}$ excitation.

\section{Cellular uptake of cRGD-MNPs}

MDA-MB-231 cells $\left(3 \times 10^{5}\right.$ per well) were seeded in 6-well plates and cultured for $24 \mathrm{~h}$. The old medium was replaced with medium containing $0.125 \mu \mathrm{M}$ cRGD-MNPs. After incubation for various times (1, 2, 4 or $8 \mathrm{~h})$, the medium was removed, and the cells were washed three times with PBS. The cells were harvested, fixed with 4\% paraformaldehyde solution for $20 \mathrm{~min}$, resuspended in PBS and counted. The same amount of cell suspension $(20 \mu \mathrm{L})$ was placed in a microcentrifuge tube and then the tube was fixed on the imaging table. PAI was performed on the MDA-MB-231 cell samples at a wavelength of $680 \mathrm{~nm}$ using the Vevo LAZR-X system. For the concentration gradient experiment, MDA-MB-231 cells were incubated in medium containing the cRGD-MNPs $(0.125,0.25,0.5$ or $1 \mu \mathrm{M})$ for $4 \mathrm{~h}$. The cells were collected and imaged as above. To investigate the effect of RGD on MNPs uptake, MDA-MB231 cells were treated for $4 \mathrm{~h}$ with medium containing $0.5 \mu \mathrm{M}$ MNPs, PEG-MNPs or cRGD-MNPs and then subjected to PAI analysis. For the RGD blocking experiment, MDA-MB-231 and MCF-10A cells were seeded in 6-well culture plates and incubated 
with $0.5 \mu \mathrm{M}$ cRGD-MNPs for $4 \mathrm{~h}$. For the $\alpha_{\mathrm{v}} \beta_{3}$ integrin-blocking group, MDA-MB231 cells were co-incubated with free RGD $(100 \mu \mathrm{M})$. The samples were assessed by PAI.

\section{Preparation of Rho-MNPs and Rho-cRGD-MNPs}

Rhodamine ( $30 \mu \mathrm{L}, 5 \mathrm{mg} / \mathrm{mL}$ ) was added to the MNPs or cRGD-MNPs solutions (1 $\mathrm{mL}, 2 \mathrm{mg} / \mathrm{mL}$ ). After stirring at room temperature for $1 \mathrm{~h}$, the solution was transferred to an ultrafiltration centrifuge tube (Millipore Amicon Ultra, $30 \mathrm{kDa}$ ) and centrifuged several times (4000 rpm, $15 \mathrm{~min}$ ) to remove the free or unstable rhodamine, yielding the rhodamine-labelled MNPs (Rho-MNPs) and cRGD-MNPs (Rho-cRGD-MNP).

\section{Location of cRGD-MNPs by confocal microscopy}

MDA-MB-231 $\left(1 \times 10^{5}\right.$ cells $)$ were seeded in confocal dishes and allowed to adhere for $24 \mathrm{~h}$. The Rho-cRGD-MNPs were added to the culture medium with or without free RGD $(100 \mu \mathrm{M})$ at the times and concentrations described above. The cells were fixed with $4 \%$ paraformaldehyde and counterstained with DAPI to visualise nuclei. The fluorescence images of the cells were collected using a Nikon confocal laser scanning microscope.

\section{In vitro cytotoxicity assay}

MNPs cytotoxicity was determined in MDA-MB-231 cells using the MTS assay. MDA-MB-231 cells $\left(5 \times 10^{3} /\right.$ well $)$ were incubated in 96-well plates for $24 \mathrm{~h}$. Cells were then cultured in medium supplemented with the different MNPs $(0.625,1.25,5$ and 10 $\mu \mathrm{M})$ for $72 \mathrm{~h}$. After the incubation, MTS $(10 \mu \mathrm{L}, 0.5 \mathrm{mg} / \mathrm{mL})$ was added to each well, and the cells were incubated for $2 \mathrm{~h}$ at $37^{\circ} \mathrm{C}$. The absorbance was measured at $490 \mathrm{~nm}$ using a microplate reader. The absorbance of the untreated cells was used as the reference value for calculating $100 \%$ cell viability. Five replicates were conducted for each group.

\section{MDA-MB-231 breast cancer xenograft mouse model}

$$
\text { MDA-MB-231 cells }\left(1 \times 10^{6} \text { in } 100 \mu \mathrm{L} \text { PBS }\right) \text { were inoculated subcutaneously in a }
$$


single flanks of nude mice. When tumour volume reached $100 \mathrm{~mm}^{3}$, the tumour-bearing mice were subjected to in vivo PAI and biodistribution studies.

\section{In vivo tumour PAI in MDA-MB-231 tumour-bearing mice}

To determine the optimal concentration range of the MNPs, mice were randomly allocated into groups and injected with different concentrations of RGD-PEG-MNPs $(50 \mu \mathrm{M}, 100 \mu \mathrm{M}$ or $200 \mu \mathrm{M}, 200 \mu \mathrm{L})$ via the tail vein. PA images were collected at different time points (pre, 1, 2, 4 or $12 \mathrm{~h}$ ) using the Vevo LAZR-X at $680 \mathrm{~nm}$, and the average PA intensities in the tumour regions were measured. To detect the tumour targeting capability of the cRGD-MNPs, MDA-MB-231 tumour-bearing mice were randomly divided into MNPs and cRGD-MNPs groups ( $\mathrm{n}=3$ per group). The mice were intravenously injected with the MNPs or cRGD-MNPs $(100 \mu \mathrm{M}, 200 \mu \mathrm{L})$, respectively. PA images were collected, and the intensities measured.

\section{The biodistribution of MNPs and cRGD-MNPs in MDA-MB-231 tumour-bearing} mice

To determine the biodistribution of the nanoprobes in vivo, MDA-MB-231 tumourbearing mice ( $\mathrm{n}=3$ per group) were intravenously injected with Rho-MNPs or RhocRGD-MNPs $(100 \mu \mathrm{M}, 200 \mu \mathrm{L})$. The major organs and tumours of mice were harvested $2 \mathrm{~h}$ post-injection for ex vivo fluorescence imaging using the IVIS Lumina imaging system (PerkinElmer, USA). The relative fluorescence intensities were measured.

\section{PAI and pathological analysis of spontaneous breast cancer in transgenic mice}

The FVB/N-Tg(MMTV-PyVT)634Mul/J transgenic mice model spontaneously develops invasive breast cancer in each mammary gland between 6 and 12 weeks of age. To further confirm the tumour targeting specificity of the cRGD-MNPs, mice with breast cancer (age 6-8 weeks, $\mathrm{n}=6$ ) were intravenously injected with the cRGD-MNPs $(100 \mu \mathrm{M}, 200 \mu \mathrm{L})$. US and PA images were obtained before injection and $2 \mathrm{~h}$ postinjection with excitation at $680 \mathrm{~nm}$. In addition, the 4th and 5th mammary glands of the transgenic mice (age 8 weeks, $\mathrm{n}=3$ ) with breast cancer were divided into four sections (P1-4) for PAI in vivo and in vitro $2 \mathrm{~h}$ after nanoprobe injection. After imaging, the 
mammary tissue was fixed with formalin and embedded in paraffin. Tissue sections (4 $\mu \mathrm{m})$ were stained with haematoxylin and eosin (H\&E), according to standard methods.

\section{Melanin staining}

The transgenic mice with breast cancer were divided into PBS and cRGD-MNPs groups (age 8 weeks, $\mathrm{n}=3$ per group). Two hours post-intravenous injection, the tumour tissue was removed and subjected to Fontana-Masson staining. The slides were immersed in the Fontana-Masson solution, placed in the dark at $4^{\circ} \mathrm{C}$ for $12-18 \mathrm{~h}$, and then rinsed with distilled water. Next, the slides was put into VG staining solution and stained for 1 minute. Finally, the slides were washed, sealed with neutral balsam and observed under the microscope.

\section{PAI-guided breast cancer surgery on MDA-MB-231 xenograft mice}

For surgical resection, cRGD-MNPs $(100 \mu \mathrm{M}, 200 \mu \mathrm{L})$ were intravenously injected into MDA-MB-231 tumour-bearing mice via the tail vein. Each mouse was anesthetised and placed on a mouse table for position adjustment. The mice remained anesthetised during the entire imaging acquisition process. Tumour profiles were detected using 3D PA/US imaging two hours after nanoprobe injection. For simulated tumour surgery resection, the surgery procedure was performed in four steps: PAI-guided tumour detection, partial tumour resection (P1-3), PAI detection of the residual tumour, and reresection of the tumour bed (P4). PA images were acquired both before and after each sequential removal of tumour. Each resection was about a 5-6 $\mathrm{mm}$ long and 2-3 $\mathrm{mm}$ wide piece of tissue. After each resection, we irrigate the surgical area with saline to resolve the bleeding issues. Surgically excised tissue was stained with haematoxylin and eosin (H\&E), according to the standard protocol. To detect the depth of PAI, tumours covering chicken breast with various thickness $(1,2,3,5,7 \mathrm{~mm})$ were performed PAI using the PAI system.

\section{cRGD-MNPs biosafety evaluation}

BALB/c mice (4-6 weeks, approximately $20 \mathrm{~g}$ ) were randomly divided into four groups: control, 1-d cRGD-MNPs, 7-d cRGD-MNPs, and 30-d cRGD-MNPs ( $\mathrm{n}=5$ per 
group). The mice were intravenously injected with saline (control) or cRGD-MNPs $(100 \mu \mathrm{M}, 200 \mu \mathrm{L})$. Body weight was monitored throughout the experiment. Blood 284 samples and major organs (heart, liver, spleen, lung and kidney) were collected after 1 (1-d group), 7 (7-d group) or 30 days (control and 30-d groups) post-injection. The blood samples were analysed for serum biochemistry parameters. These parameters included aspartate aminotransferase (AST), alanine aminotransferase (ALT), blood urea nitrogen (BUN) and creatinine (CR). H\&E staining was performed on sections of the major organs for histological analysis.

\section{0}

\section{Statistical analysis}

291 Statistical analysis was performed using GraphPad Prism 7.0 (GraphPad Software, 292 CA). The two-tailed paired t-test was used to compare the changes in photoacoustic 293 signals. Data are presented as the mean \pm standard error of the mean. Significant 294 differences between or among the groups are indicated as follows: ns for no significant 295 difference, $*$ for $P<0.05, * *$ for $P<0.01$, and $* * *$ for $P<0.001$. 


\section{Results}

298

299

300

301

302

303

304

305

306

307

\section{Synthesis and characterisation of cRGD-MNPs}

The water-soluble MNPs were approximately $4 \mathrm{~nm}$ in size based on transmission electron microscopy (Fig. 1a). Polyethylene glycol (PEG) chains were introduced into the MNPs for further biomodification, which was confirmed by ${ }^{1} \mathrm{H}$ NMR spectra (Fig. S1a). This can reduce the liver accumulation because of the enhanced water solubility. Lastly, the PEG-MNPs were further modified with cyclic Arg-Gly-Asp-d-Phe-Cys [c(RGDfC)] peptide (cRGD), which targets tumour $\alpha_{\mathrm{v}} \beta_{3}$ integrin [21]. The diameter of the RGD-functionalized PEG-MNPs (cRGD-MNPs) increased to about $9 \mathrm{~nm}$ (Fig. 1a). With the modifications of PEG and RGD (i.e., cRGD-MNPs), the surface potential of the MNPs increased from -48 to $-8.6 \mathrm{mV}$ (Fig. 1b). MNPs have strong and broad optical absorbance and PA spectra in the wavelength range of visible light to the near-infrared region. The similar absorption and PA spectra between cRGD-MNPs and MNPs indicated that the biomodifications did not influence the absorption properties and intrinsic PA properties of melanin (Fig. 1c, d). Moreover, both the cRGD-MNPs and MNPs showed a linear relationship between concentration and absorbance intensity $\left(\mathrm{R}^{2}\right.$ $=1$ and 0.9999 for MNPs and cRGD-MNPs, respectively) (Fig. S1b, c, d) and PA intensity $\left(\mathrm{R}^{2}=0.9637\right.$ and 0.9513 for MNPs and cRGD-MNPs, respectively) (Fig. S1e, f, Fig. 1e) at $680 \mathrm{~nm}$. In addition, the cRGD-MNPs exhibited excellent stability, with no attenuation of the PA signal intensity after storage for a week at $4^{\circ} \mathrm{C}$ (Fig. 1f).

\section{Targeting ability and cytotoxicity of cRGD-MNPs in vitro}

As seen from the overlay images and quantitative results, the PA signal intensity of MDA-MB-231 cells increased with incubation time from $1 \mathrm{~h}$ to $8 \mathrm{~h}$ (Fig. 2a). The cells produced significant PA signal intensities after incubation for $4 \mathrm{~h}$, and there was no statistical difference between the $4 \mathrm{~h}$ and $8 \mathrm{~h}$ time points. As the concentration of cRGDMNPs increased, the uptake of the probe by the MDA-MB-231 cells also increased (Fig. 2b). The concentration of $0.5 \mu \mathrm{M}$ was selected for subsequent experiments because there was no statistical difference between $0.5 \mu \mathrm{M}$ and $1 \mu \mathrm{M}$ in PA signal intensity. 
Compared to the same concentrations of MNPs and PEG-MNPs, MDA-MB-231 cells incubated with RGD-MNPs showed stronger PA signal intensity (Fig. 2c), which confirmed that more cRGD-MNPs could be internalised by MDA-MB-231 cells.

To prove the ability of the cRGD-MNPs to target the integrin $\alpha_{\mathrm{v}} \beta_{3}$ receptor, we determined their binding affinity using MDA-MB-231 and MCF-10A (a non-neoplastic breast cell line with low expression of $\alpha_{v} \beta_{3}$ ) [22] cells. The PA intensity was markedly enhanced for the MDA-MB-231 cells compared to that of the MCF-10A cells after a 4$\mathrm{h}$ incubation (Fig. 2d). Additionally, when the cells were co-incubated with excess free RGD molecules, the PA signal intensity of the MDA-MB-231 cells became weaker. These data indicated that indeed RGD enhanced the internalisation of the nanoparticles by cancer cells overexpressing the integrin $\alpha_{v} \beta_{3}$ receptor. Similarly, strong red fluorescence was observed in MDA-MB-231 cells incubated with Rho-cRGD-MNPs, while MCF-10A cells showed very weak red fluorescence under the same conditions. This phenomenon could be blocked by excessive unlabelled RGD peptide (Fig. 2e).

Finally, analysis of cell viability suggested low cytotoxicity for the MNPs, even at a high concentration up to $10 \mu \mathrm{M}$ (Fig. 2f).

\section{In vivo cRGD-MNPs tumour uptake and the biodistribution of fluorescence- labelled cRGD-MNPs}

Based on the PAI performance of the cRGD-MNPs in vitro, we performed tumour PAI in vivo. The in vivo PA signal intensity at the tumour site of MDA-MB-231 tumourbearing mice at various concentration of cRGD-MNPs $(50,100$, and $200 \mu \mathrm{M})$ increased gradually and reached a peak two hours post-injection (Fig. S2a). Moreover, the 100 $\mu \mathrm{M}$ and $200 \mu \mathrm{M}$ groups exhibited similar PA intensities, which were higher than that observed for the $50 \mu \mathrm{M}$ group $2 \mathrm{~h}$ post-injection. The tumour targeting capability of the cRGD-MNPs in living mice was further investigated by intravenous injection cRGDMNPs into tumour-bearing mice using MNPs as a control) (Fig. 3a, b). We showed that tumour sites exhibited a higher PA signal 1 to $2 \mathrm{~h}$ after intravenous administration of cRGD-MNPs than MNPs in vivo. Moreover, the cRGD-MNPs group had a significantly higher signal-to-background ratio (tumour versus surrounding normal tissue) than the 
MNPs group two hours post-injection $(3.2 \pm 0.1$ versus $1.7 \pm 0.3, P<0.05)$ (Fig. $3 \mathrm{c}$ ). In addition, the PA signal increased approximately 2.1-fold two hours post-injection compared to pre-injection (Fig. S2b). These results indicated that cRGD-MNPs accumulated more in tumours than normal tissue and provided clearer tumour contrast. We also used fluorescence imaging to track the biodistribution of Rho-cRGD-MNPs and Rho-MNPs two hours post-injection (Fig. 3d). The ex vivo fluorescence images and intensities of the major organs and tumours suggested more effective accumulation of Rho-cRGD-MNPs into tumour tissue than Rho-MNPs (Fig. 3d, e).

\section{In vivo cRGD-MNPs PAI in MMTV-PyVT transgenic mice}

To evaluate the PAI performance of cRGD-MNPs, we used the FVB/N-Tg(MMTVPyVT)634Mul/J spontaneous breast cancer model that closely recapitulate human disease [23]. The transgenic mice underwent PAI before or two hours post-injection (Fig. 4a). Compared to the imaging signal before administration, the PA signal intensities of the mammary glands containing tumour increased $2.5 \pm 0.3$-fold (Fig. 4b). In contrast, the PA signal intensities of the normal mammary glands did not increase post-injection $(0.9 \pm 0.1$-fold, Fig. $4 \mathrm{~b})$. The pathological status of the mammary glands was confirmed by H\&E staining (Fig. 4c). Furthermore, melanin staining confirmed the presence of the MNPs in the tumour tissue after injection of the probe (Fig. 4d). These results indicate that cRGD-MNPs provide a high signal intensity at the tumour site and could distinguish between normal mammary glands and breast tumours.

In order to further assess the feasibility of using the cRGD-MNPs to detect a tumour, segmented tumour PAI was performed in MMTV-PyVT transgenic mice with breast cancer (Fig. 4e). The fourth and fifth mammary glands of the transgenic mice were divided into four segments (P1-4), and PA imaging showed complete and intense enhancement of each part of the tumour. Ex vivo tissues were imaged using the PAI system, and the tissue signal distribution was consistent with that observed in vivo and correlated with the pathological examination (Fig. S3a). The PA images demonstrated 
an improved contrast profile for breast cancer detection with the cRGD-MNPs.

383

384

385

386

387

388

389

390

391

392

393

394

395

396

397

398

399

400

401

402

403

404

405

406

407

408

409

\section{PAI-guided resection of breast cancer using cRGD-MNPs}

We evaluated cRGD-MNPs-based PAI for tumour detection, delineation, and imaging-guided resection. First, the tumours of MDA-MB-231 tumour-bearing mice were examined by PAI preoperatively. The reconstructed three-dimensional (3D) image was used to show the spatial distribution of the cRGD-MNPs at the tumour site, which provided the general profile of a tumour for the development of a surgical plan (Fig. 5a). From the PA signals of the cRGD-MNPs, the size and position of the MDA-MB231-tumour could be defined.

Next, we investigated whether the nanoprobes could guide intraoperative tumour resection. In this experiment, MDA-MB-231 tumour-bearing mice underwent consecutive tumour resections two hours post-injection of cRGD-MNPs. Ultrasound (US) images depicted representative subcutaneous transplanted tumours (Fig. 5b). A strong PA signal showed the tumour location before the resection. We then performed partial resection under PAI guidance. The resected tissues for each step are shown in Figure $5 \mathrm{~b}(\mathrm{P} 1-4)$. After removal of most of the tumour, the remaining tumour (about 2 mm wide) was still visible by PAI. Finally, the remaining tumour was resected until no obvious PA signal was detected. For comparison, tissues with negative PA signals on the tumour bed (P4) were excised again for subsequent pathology examination. The ex vivo tissue PA signal distribution was consistent with that obtained in vivo (Fig. S3b). Pathological examination showed that the tumour had been completely removed, and the tissue with a negative PA signal on the tumour bed was muscle tissue (Fig. 5c). To detect the depth of PAI, we covered the tumours with chicken breasts of various thicknesses and found that the maximum imaging depth was up to $5 \mathrm{~mm}$ (Fig. 5d). Taken together, PAI can effectively detect tumours, identify residual masses, and guide surgical resection.

\section{In vivo cRGD-MNPs biosafety}

The in vivo biocompatibility was further evaluated in mice for prolonged durations 
410 (1, 7 or 30 days) after intravenous administration of cRGD-MNPs. Saline served as a

411 negative control. No considerable body weight loss was observed for the cRGD-MNPs

412 and control groups over 30 days, indicating that cRGD-MNPs had no significant side

413 effects in mice (Fig. 6a). The serum biochemistry analysis, which included liver

414 function (ALT, AST) and kidney function (BUN, CR) markers, showed negligible

415 variations between the different groups, indicating no detectable toxicity shortly after

416 or a relatively long time after exposure (Fig. 6b). Although cRGD-MNPs also

417 accumulated in the liver and kidneys, negligible liver and kidney damage was induced.

418 H\&E staining of the major organs (heart, liver, spleen, lung and kidney) at different

419 times after intravenous injection showed no significant acute or chronic physiological

420 toxicity compared to the control group, indicating the high histocompatibility of the

421 cRGD-MNPs (Fig. 6c). These results indicate that the cRGD-MNPs have high in vivo

422 biocompatibility. 


\section{Discussion}

It remains a significant challenge in BCS to acquire clean margins during primary surgery due to the lack of precision in localising tumours and the inaccuracy of tumour excision by visual inspection and tactile feedback. Imaging-guided surgery is gaining increasing importance in the operating room. It can help detect microscopic tumours or residual lesions that are readily missed during surgery and guide intraoperative surgical margin assessment [24]. Thus, this approach could potentially improve patient outcome following oncologic surgery. In the present study, we demonstrated for the first time that that the use of cRGD-MNPs as a targeted PA contrast has the potential to locate tumours and offer a 3D reconstruction of the breast cancer for surgical planning. Although the utility of cRGD-MNPs-mediated PAI or photothermal therapy has been previously demonstrated in animals [17], our study further translates these findings to imaging-guided cancer surgery, showing the feasibility of cRGD-MNPs PAI for consecutive imaging-guided resections of the remaining margins of breast cancer.

Melanin is a naturally occurring biopolymer present in many organisms, particularly in the skin and hair. It has a good intrinsic photoacoustic property. By mimicking natural melanin, recent studies have demonstrated that melanin-based nanoparticles could serve as a multimodality nanoplatform for molecular imaging [16, 25, 26]. As in the report of Fan et al [20], we prepared artificial ultrasmall MNPs and modified them by conjugating them with $\mathrm{c}(\mathrm{RGDFC})$ peptides. cRGD-MNPs have a high affinity for integrin $\alpha_{\mathrm{v}} \beta_{3}$, which is expressed predominantly on neovascular endothelial cells and tumour cells, including breast cancer cells [27, 28]. Consistent with previous findings, our results demonstrated a higher tumour-targeting capability of cRGD-MNPs compared to MNPs using MDA-MB-231 tumour-bearing mice, indicating that cRGDMNPs could more specifically target breast cancer xenografts, possibly via the binding to integrin $\alpha_{\mathrm{v}} \beta_{3}$. Moreover, mammary glands of FVB/N-Tg(MMTV-PyVT)634Mul/J transgenic mice containing carcinoma showed excellent PA intensity enhancement after the administration of cRGD-MNPs compared to the normal mammary gland tissue. These data further indicated that cRGD-MNPs could conveniently serve as a good contrast for breast cancer-specific PAI. 
MNPs have been used in diverse biomedical applications, such as imaging, controlled drug release, bioengineering and bioelectronics, antioxidant applications and theranostics [29]. Based on the dramatic in vivo PAI properties of the cRGD-MNPs in this study, we used these MNPs for PAI-guided surgical navigation. To simulate PAIguided consecutive resections in a small animal model, we sequentially removed breast tumour tissue using the real-time guidance of PAI, which was validated by subsequent histological analysis. In addition, we found that the tumour, when artificially located in deep tissue, could be clearly imaged and discriminated with a depth of up to $5 \mathrm{~mm}$. A previous study reported the application of PAI with endogenous melanin as contrast for the resection of B16 melanoma liver metastasis [30]. Hepatic melanoma in vivo as small as $400 \mu \mathrm{m}$ could be detected at a depth of up to $7 \mathrm{~mm}$ and precisely resected using PAI guidance, demonstrating the advantages of PA (i.e., high resolution, high sensitivity, deep penetration and early detection of hepatic micrometastasis). In a clinical study, PAI-guided pathological evaluation improved the detection rate of metastases compared to the standard protocol in excising sentinel lymph nodes in patients with melanoma (22.9\% versus $14.2 \%$ ) [19]. Taken together with the results of the present study, PAI using melanin or melanin-based nanoparticles could offer a rapid and effective tool for non-invasive detection of small tumour disease of a certain depth.

In addition to melanin nanoparticles, several exogenous contrast agents have been used for photoacoustic surgical navigation in animals, including gold nanoparticles [31, 32], superparamagnetic iron oxide [33], dye-based agents [34, 35], and carbon-based nanomaterials [36]. All these agents could generate non-invasive PA contrast enhancement when stimulated by laser irradiation at specific wavelengths. However, clinical translation of these agents is prohibitive because of biosafety issues, poor biodegradability, low photostability and unclear biocompatibility [37, 38]. In the present study, the biocompatibility and biosafety of cRGD-MNPs were systematically evaluated both in vitro and in vivo. As reported previously, the strengths of melaninlike nanomaterials include good biocompatibility and long-term photostability [39, 40], prompting us to explore their biomedical applications, particularly for in vivo imaging. 
further clinical translation.

However, this study has a few limitations. First, only PAI alone was applied to surgical navigation. As a previous study reported [20], MNPs are an active platform to simplify the assembling of different imaging moieties, such as positron emission tomography and magnetic resonance imaging. Thus, complementary use of multimodality imaging is promising not only for accurate tumour imaging but also for guiding tumour resection. Further research efforts should be devoted to precise, targeted tumour multimodality imaging. Second, the current methodology using MNPs as a contrast agent is unsuitable for deep-tissue imaging in the human body. Therefore, further improvements of the imaging agents are needed to increase the imaging depth. In this report, we demonstrated the feasibility of PA augmented by the systemic delivery of cRGD-MNPs to detect tumours for preoperative 2D or 3D imaging and guide initial and subsequent resections of breast cancer xenografts. With further development and optimisation, PAI using cRGD-MNPs is expected to be evaluated in breast cancer patients for surgical navigation in the near future. 


\section{References}

500 1. DeSantis C E, Ma J, Gaudet M M, Newman L A, Miller K D, Goding Sauer A, et al. Breast cancer statistics, 2019. CA: a cancer journal for clinicians. 2019; 69:438-451. DOI: 10.3322/caac.21583.

Darby S, McGale P, Correa C, Taylor C, Arriagada R, Clarke M, et al. Effect of radiotherapy after breastconserving surgery on 10-year recurrence and 15-year breast cancer death: meta-analysis of individual patient data for 10,801 women in 17 randomised trials. Lancet. 2011; 378:1707-1716. DOI: 10.1016/S0140-6736(11)61629-2. Pleijhuis R G, Graafland M, de Vries J, Bart J, de Jong J Svan Dam G M. Obtaining adequate surgical margins in breast-conserving therapy for patients with early-stage breast cancer: current modalities and future directions. Annals of surgical oncology. 2009; 16:2717-2730. DOI: 10.1245/s10434-009-0609-z.

5. Pradipta A R, Tanei T, Morimoto K, Shimazu K, Noguchi STanaka K. Emerging Technologies for RealEsbona K, Li ZWilke L G. Intraoperative imprint cytology and frozen section pathology for margin Time Intraoperative Margin Assessment in Future Breast-Conserving Surgery. Advanced science. 2020; 7:1901519. DOI: $10.1002 /$ advs.201901519.

9. Hernot S, van Manen L, Debie P, Mieog J S DVahrmeijer A L. Latest developments in molecular tracers

10. Nguyen Q TTsien R Y. Fluorescence-guided surgery with live molecular navigation--a new cutting edge.

11. Wang L VYao J. A practical guide to photoacoustic tomography in the life sciences. Nature Methods. 2016;

12. Li R, Lan L, Xia Y, Wang P, Han L K, Dunnington G L, et al. High-speed Intraoperative Assessment of

13. Wong T T W, Zhang R, Hai P, Zhang C, Pleitez M A, Aft R L, et al. Fast label-free multilayered histologyphotoacoustic tomography. Biomedical optics express. 2015; 6:1273-1281. DOI: 10.1364/BOE.6.001273. Weber J, Beard P CBohndiek S E. Contrast agents for molecular photoacoustic imaging. Nature Methods. 2016; 13:639-50. DOI: 10.1038/nmeth.3929. 
16. Park J, Moon HHong S. Recent advances in melanin-like nanomaterials in biomedical applications: a mini review. Biomaterials research. 2019; 23:24-24. DOI: 10.1186/s40824-019-0175-9.

17. Caldas M, Santos A C, Veiga F, Rebelo R, Reis R LCorrelo V M. Melanin nanoparticles as a promising

18. Fu Q, Zhu R, Song J, Yang HChen X. Photoacoustic Imaging: Contrast Agents and Their Biomedical

19. Stoffels I, Morscher S, Helfrich I, Hillen U, Leyh J, Lehy J, et al. Metastatic status of sentinel lymph nodes

20. Fan Q, Cheng K, Hu X, Ma X, Zhang R, Yang M, et al. Transferring biomarker into molecular probe:

21. Nieberler M, Reuning U, Reichart F, Notni J, Wester H J, Schwaiger M, et al. Exploring the Role of RGD-

22. Veneti E, Tu R SAuguste D T. RGD-Targeted Liposome Binding and Uptake on Breast Cancer Cells Is

23. Shishido S N, Delahaye A, Beck ANguyen T A. The anticancer effect of PQ1 in the MMTV-PyVT mouse model. International Journal of Cancer. 2014; 134:1474-1483. DOI: 10.1002/ijc.28461.

25. Liu H, Yang Y, Liu Y, Pan J, Wang J, Man F, et al. Melanin-Like Nanomaterials for Advanced Biomedical 10.1002/advs.201903129. of Cancer. Theranostics. 2018; 8:1591-1606. DOI: 10.7150/thno.22430.

Zhang L, Sheng D, Wang D, Yao Y, Yang K, Wang Z, et al. Bioinspired Multifunctional Melanin-Based Zhang J, Mao F, Niu G, Peng L, Lang L, Li F, et al. Ga-BBN-RGD PET/CT for GRPR and Integrin $\alpha \beta$ Imaging in Patients with Breast Cancer. Theranostics. 2018; 8:1121-1130. DOI: 10.7150/thno.22601.

Beer A J, Niemeyer M, Carlsen J, Sarbia M, Nährig J, Watzlowik P, et al. Patterns of alphavbeta3 expression in primary and metastatic human breast cancer as shown by $18 \mathrm{~F}-$ Galacto-RGD PET. Journal of nuclear medicine. 2008; 49:255-259. DOI: 10.2967/jnumed.107.045526.

Hong Z-Y, Feng H-YBu L-H. Melanin-based Nanomaterials: The promising Nanoplatforms for Cancer diagnosis and therapy. Nanomedicine. 2020:102211. DOI: 10.1016/j.nano.2020.102211. Micrometastasis and Intraoperative Image-guided Surgery by Photoacoustic Imaging. Journal of nuclear medicine. 2019; 61:1079-1085. DOI: 10.2967/jnumed.119.233155.

Kircher M F, de la Zerda A, Jokerst J V, Zavaleta C L, Kempen P J, Mittra E, et al. A brain tumor molecular imaging strategy using a new triple-modality MRI-photoacoustic-Raman nanoparticle. Nature medicine. 2012; 18:829-834. DOI: 10.1038/nm.2721.

32. Guan T, Shang W, Li H, Yang X, Fang C, Tian J, et al. From Detection to Resection: Photoacoustic 
Tomography and Surgery Guidance with Indocyanine Green Loaded Gold Nanorod@liposome Core-Shell Nanoparticles in Liver Cancer. Bioconjugate chemistry. 2017; 28:1221-1228. DOI: 10.1021/acs.bioconjchem.7b00065.

33. Thawani J P, Amirshaghaghi A, Yan L, Stein J M, Liu JTsourkas A. Photoacoustic-Guided Surgery with

35. Han Z, Shang W, Liang X, Yan H, Hu M, Peng L, et al. An Innovation for Treating Orthotopic Pancreatic

36. De la Zerda A, Zavaleta C, Keren S, Vaithilingam S, Bodapati S, Liu Z, et al. Carbon nanotubes as Indocyanine Green-Coated Superparamagnetic Iron Oxide Nanoparticle Clusters. Small. 2017; 13. DOI:

34. Wilson K E, Bachawal S VWillmann J K. Intraoperative Resection Guidance with Photoacoustic and Fluorescence Molecular Imaging Using an Anti-B7-H3 Antibody-Indocyanine Green Dual Contrast Agent. Clinical cancer research : an official journal of the American Association for Cancer Research. 2018; photoacoustic molecular imaging agents in living mice. Nature nanotechnology. 2008; 3:557-62. DOI: 10.1038/nnano.2008.231

37. Zhang X-D, Wu D, Shen X, Liu P-X, Yang N, Zhao B, et al. Size-dependent in vivo toxicity of PEGcoated gold nanoparticles. International journal of nanomedicine. 2011; 6:2071-2081. DOI: 10.2147/IJN.S21657.

8. Bussy C, Methven LKostarelos K. Hemotoxicity of carbon nanotubes. Advanced Drug Delivery Reviews. 2013; 65:2127-2134. DOI: 10.1016/j.addr.2013.10.008.

Liu Y, Ai K, Liu J, Deng M, He YLu L. Dopamine-melanin colloidal nanospheres: an efficient nearinfrared photothermal therapeutic agent for in vivo cancer therapy. Advanced materials. 2013; 25:13531359. DOI: 10.1002/adma.201204683.

613 


\section{Declarations}

616

617

618

619

620

621

622

623

624

625

626

627

628

629

630

631

632

633

634

635

636

637

\section{Funding}

The present study was supported by the National Natural Science Foundation of China (No. 91859120), Natural Science Foundation of Guangdong Province (No. 2016A030312008), Scientific Research Fund of Traditional Chinese Medicine Bureau of Guangdong Province (No. 20181146), Start-Up Fund for High-Talents from Xiang'an Hospital of Xiamen University (No. PM201809170013).

\section{Conflict of interest}

The authors declare that they have no conflicts of interest.

\section{Data availability}

The datasets generated during and/or analysed during the current study are available from the corresponding author on reasonable request.

\section{Contributor Information}

Jing-Jing Liu conducted experiments and wrote the manuscript. Zun Wang coordinated and performed the study and drafted the manuscript. Li-Ming Nie directed the experimental methods. Yuan-Yuan Zhu, Ge Li and Lin-Ling Lin cultured cells, raised mice and conducted experiments. Min Chen developed the experimental design and directed the study. Guo-Jun Zhang designed and directed the study and finalised the manuscript.

\section{Ethical approval}

Approval was obtained from the ethics committee of Xiamen University. The procedures used in this study adhere to the tenets of the Declaration of Helsinki. 


\section{Figure Legends}

Scheme 1 Workflow of cRGD-MNPs for intraoperative imaging-guided surgery by photoacoustic imaging.

Fig. 1 Characterisation of cRGD-MNPs. a Representative transmission electron microscopy images of MNPs (left) and cRGD-MNPs (right), scale bar: $20 \mathrm{~nm}$. b Zeta potential of MNPs, PEG-MNPs and cRGD-MNPs in aqueous solution. $\mathbf{c}$ UV-vis-NIR spectra of MNPs and cRGD-MNPs. d PA spectra of MNPs and cRGD-MNPs. e The photoacoustic signal produced by MNPs and cRGD-MNPs was linear at concentrations from 3.75 to $120 \mu \mathrm{M}$. Upper panel: the original PA image. f PA signal intensities of cRGD-MNPs in PBS ( $\mathrm{pH}=7.4$ ) stored for one week. Data are presented as the mean \pm $\operatorname{SD}(n=3)$.

Fig. 2 In vitro uptake and cytotoxicity of cRGD-MNPs. a PA images and signal intensities of MDA-MB-231 cells incubated with cRGD-MNPs for various times $(0,1$, 2, 4 and 8 h). b PA images and signal intensities of MDA-MB-231 cells incubated with cRGD-MNPs at various concentrations $(0,0.125,0.25,0.5$ and $1 \mu \mathrm{M})$. c PA images and signal intensities of MDA-MB-231 cells incubated with $0.5 \mu \mathrm{M}$ MNPs, PEG-MNPs or cRGD-MNPs for $4 \mathrm{~h}$. PBS was used as a negative control. d PA images and signal intensities of MCF-10A and MDA-MB-231 cells after incubation with cRGD-MNPs for $4 \mathrm{~h}$ with or without free RGD blocking. e CLSM images of MCF-10A and MDAMB-231 cells incubated with Rho-cRGD-MNPs for $4 \mathrm{~h}$ with or without free RGD blocking. Scale bar: $20 \mu \mathrm{m}$. f MDA-MB-231 cell viability after incubation with MNPs, PEG-MNPs or cRGD-MNPs at gradient concentrations using standard MTS assay. Data are presented as the mean $\pm \mathrm{SD}(\mathrm{n}=) 3, * * * P<0.001$.

Fig. 3 Tumour-targeting and biodistribution of cRGD-MNPs using an MDA-MB231 xenograft mouse model. a In vivo merged PA and US images of MDA-MB-231 tumour-bearing mice at various times (pre, 1, 2, 4 and $12 \mathrm{~h}$ ) after intravenous injection 
of MNPs or cRGD-MNPs $(100 \mu \mathrm{M}, 200 \mu \mathrm{L})$. Scale bar: $2 \mathrm{~mm}$. b Quantitative analysis of PA intensities of tumour sites at the different time points in (a). c Signal-tobackground ratio of the tumour region two hours post-injection of MNPs or cRGDMNPs. $\mathbf{d}$ The biodistribution of rhodamine-labelled MNPs or cRGD-MNPs in the heart, liver, spleen, lung, kidney and tumour from MDA-MB-231 tumour-bearing mice two hours post-injection. e Quantitative analysis of the fluorescence intensities of the tissues in $(\mathbf{d})$. Data are presented as the mean $\pm \mathrm{SD}(\mathrm{n}=3) ; * * * P<0.001, * P<0.05$.

\section{Fig. 4 PAI for mammary glands containing spontaneous breast cancer in MMTV-} PyVT transgenic mice. a Representative US and PA images of cRGD-MNPs accumulation in mice with normal mammary glands or breast cancer. Scale bar: $2 \mathrm{~mm}$. b PA signal intensities of breast tumours and normal mammary glands in transgenic mice two hours after tail-vein injection of cRGD-MNPs compared to $0 \mathrm{~h}$. c Histopathological examination of the tissues from (a). Scale bar: $2 \mathrm{~mm}$. d FontanaMasson staining of ex vivo tumour tissue. Black particles representing the MNPs were not observed in the non-injected nanoprobe group (left). Black particles representing the cRGD-MNPs were observed in the injection probe group (right). The image in the lower right corner (black square) is an enlarged image of the upper left corner area. Scale bar: $200 \mu \mathrm{m}$. e US (top), PA (middle) and histological (bottom) images of the 4th and 5th pairs of mammary glands in an 8-week-old MMTV-PyVT mouse. The enlarged mammary glands and different regional tissue (P1-4) are outlined with dotted orange and green lines, respectively. The dotted blue line outlines the tissue inferior to the mammary gland. Scale bar: $2 \mathrm{~mm}$. Data are presented as the mean $\pm \mathrm{SD}(\mathrm{n}=3) ; * P<$ 0.05 .

\section{Fig. 5 PAI-guided tumour resection in MDA-MB-231 tumour-bearing mice. a} Render, axial, sagittal and coronal images showing the tumour distribution. Scale bar: $2 \mathrm{~mm}$. b Anatomical US (top) and PA (bottom) images showing the tumour region (dotted orange line) in MDA-MB-231 tumour-bearing mice in vivo. The tissue portion to be resected (P1-4) is highlighted with a green dashed circle. Scale bar: $2 \mathrm{~mm}$. c 
697 Histological images (top) of resected tissue pieces. Scale bar: $2 \mathrm{~mm}$. Enlarged images 698 of regions marked with black boxes are also shown (bottom). Scale bar: $100 \mu \mathrm{m}$. d PA 699 and US images of tumour tissues covered by different thicknesses of chicken breast 700 under $680 \mathrm{~nm}$ laser excitation in vitro. Scale bar: $2 \mathrm{~mm}$.

701

Fig. 6 The biosafety of cRGD-MNPs. a Body weight curves for mice treated 703 intravenously with saline (control) or cRGD-MNPs. b Serum biochemistry results 704 (liver function and renal function) for BALB/c mice at various time points $(1,7$ and 30 705 days) after intravenous injection of cRGD-MNPs or saline (controls). The units of ALT 706 and AST are $\mathrm{U} / \mathrm{L}$; the units of BUN and CR are $\mathrm{mmol} / \mathrm{L}$ and $\mu \mathrm{mol} / \mathrm{L}$, respectively. c 707 H\&E staining of vital organs (heart, liver, spleen, lung and kidney) from each group. Scale bar: $200 \mu \mathrm{m}$. Data are presented as the mean \pm SD $(n=5)$. 
Figures

a
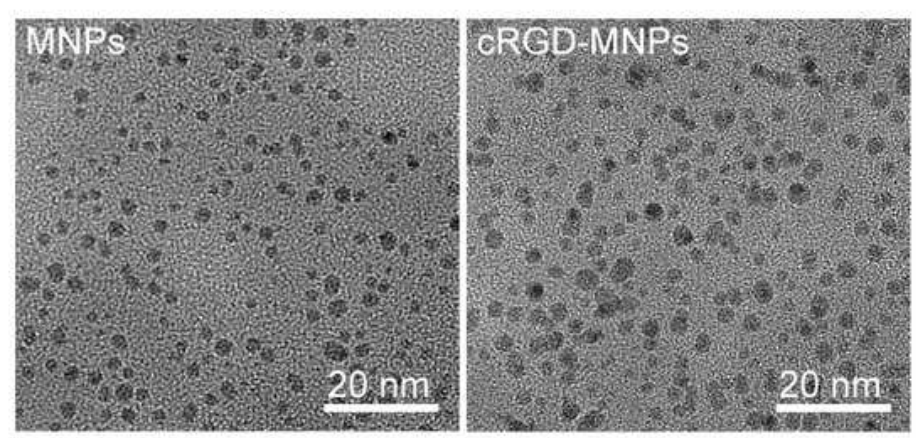

C

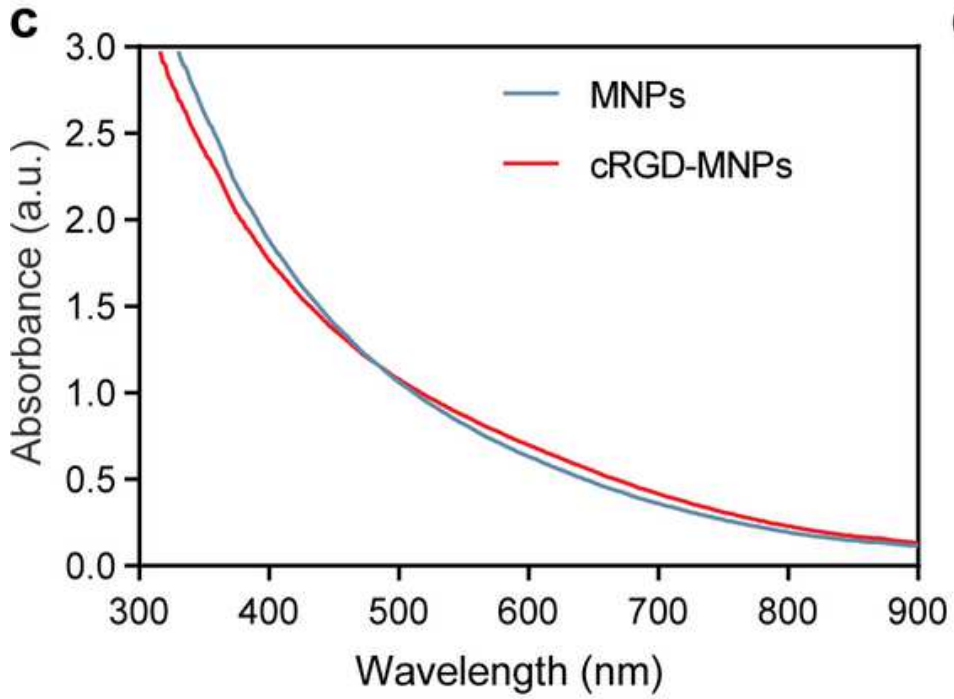

e

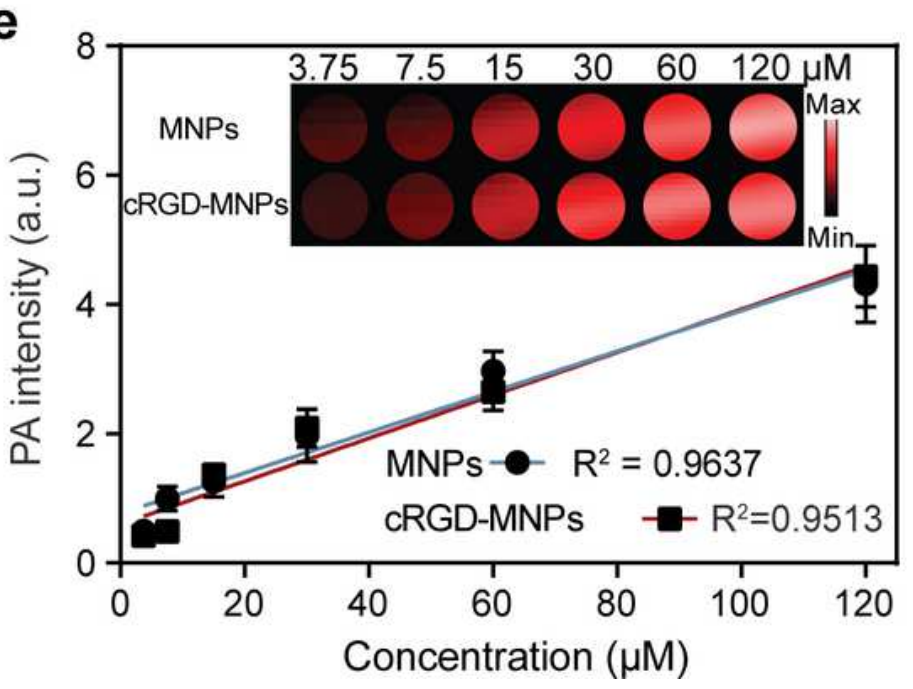

b

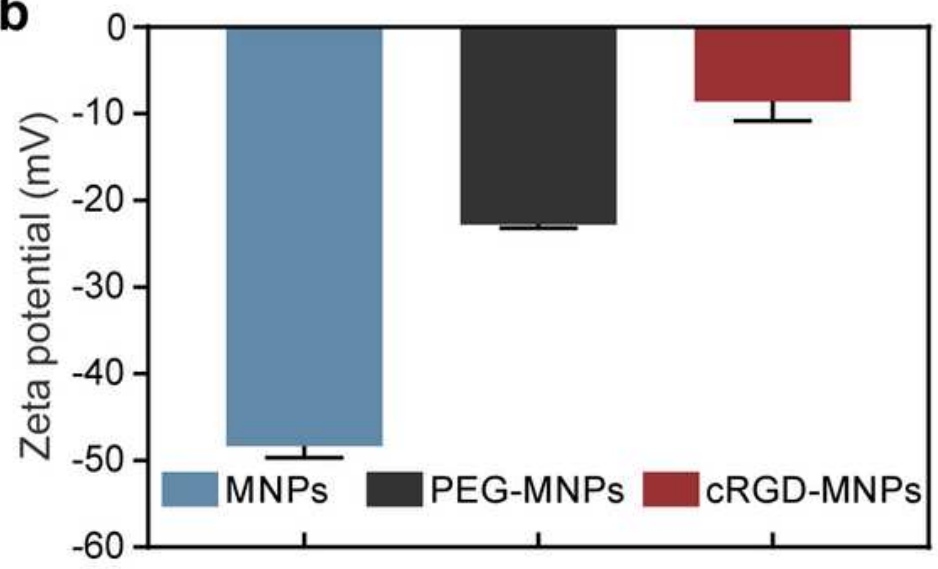

d

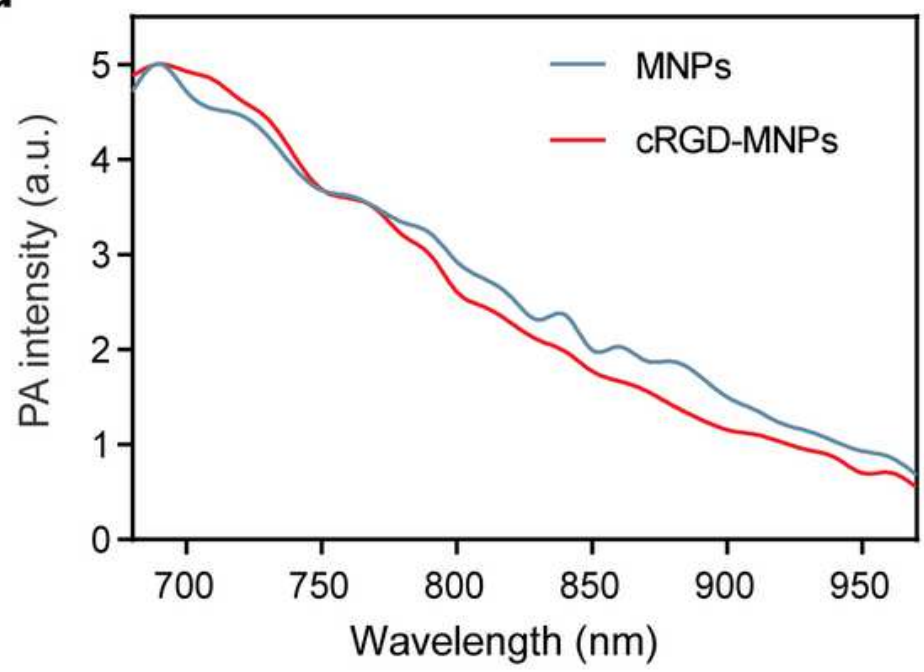

f

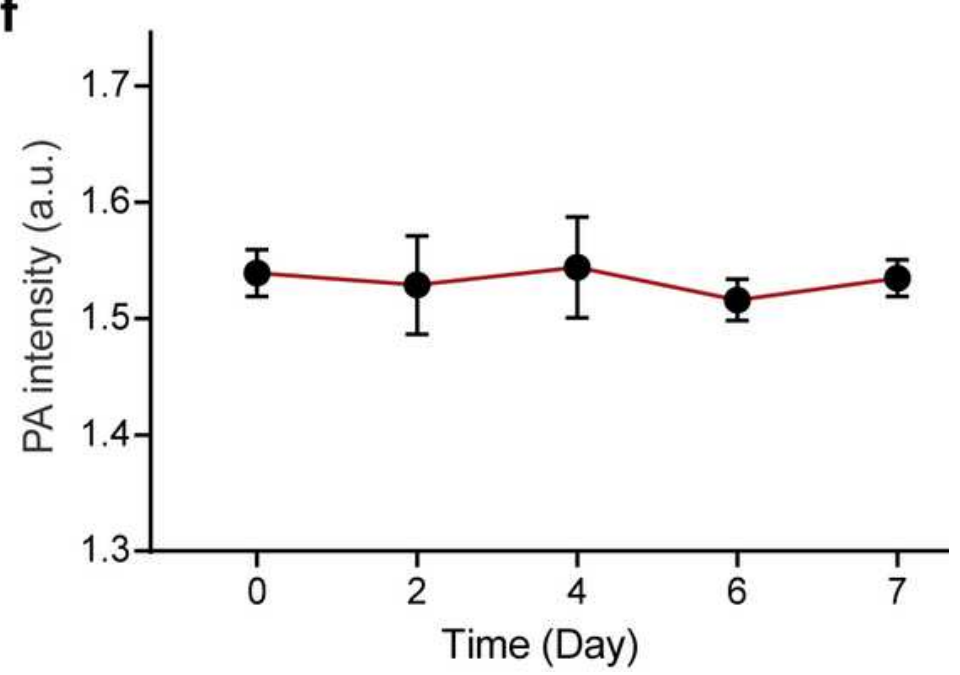

Figure 1

Characterisation of cRGD-MNPs. a Representative transmission electron microscopy images of MNPs (left) and cRGD-MNPs (right), scale bar: $20 \mathrm{~nm}$. b Zeta potential of MNPs, PEG-MNPs and cRGD-MNPs in aqueous solution. c UV-vis-NIR spectra of MNPs and cRGD-MNPs. d PA spectra of MNPs and cRGD- 
MNPs. e The photoacoustic signal produced by MNPs and cRGD-MNPs was linear at concentrations from 3.75 to $120 \mu \mathrm{M}$. Upper panel: the original PA image. f PA signal intensities of cRGD-MNPs in PBS $(\mathrm{pH}=7.4)$ stored for one week. Data are presented as the mean \pm SD $(n=3)$.

a

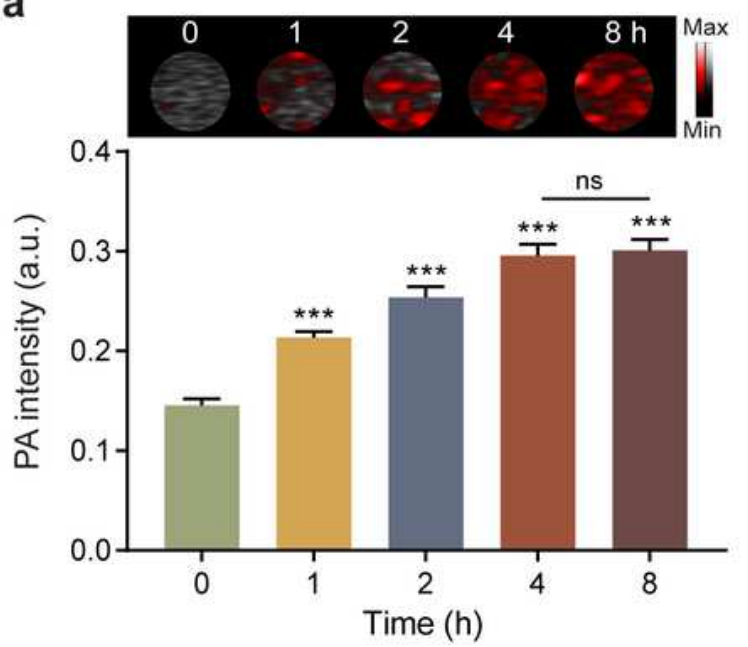

C

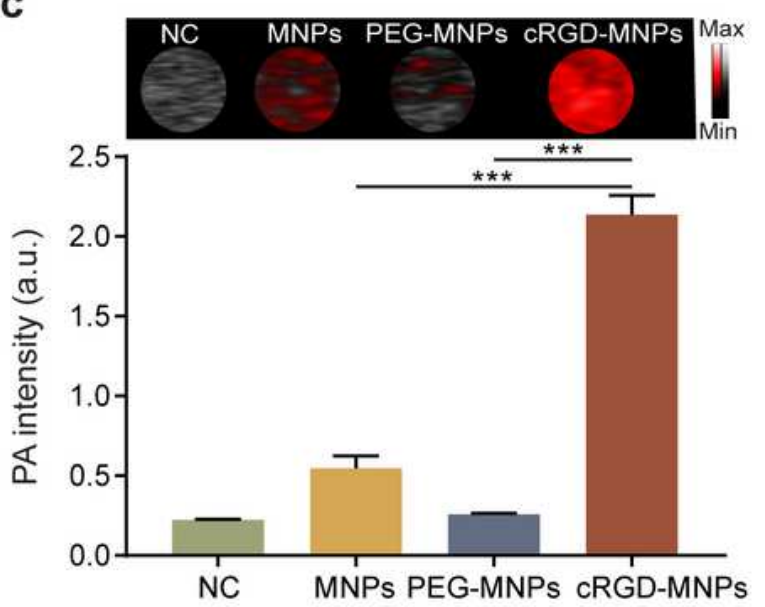

e

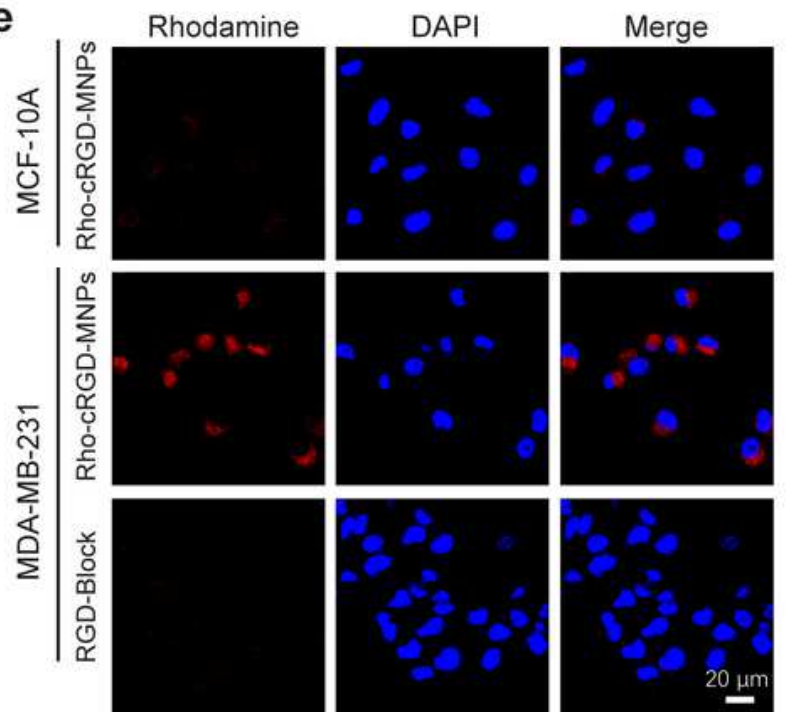

b

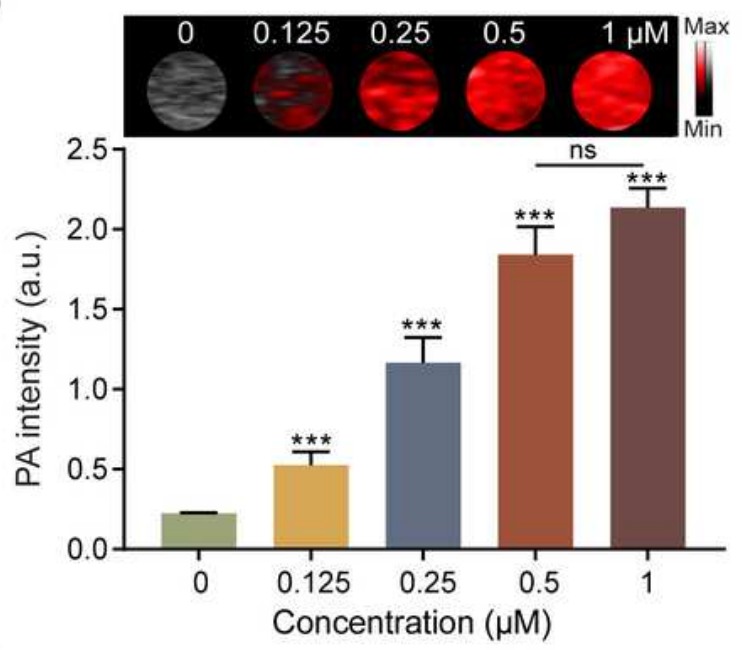

d

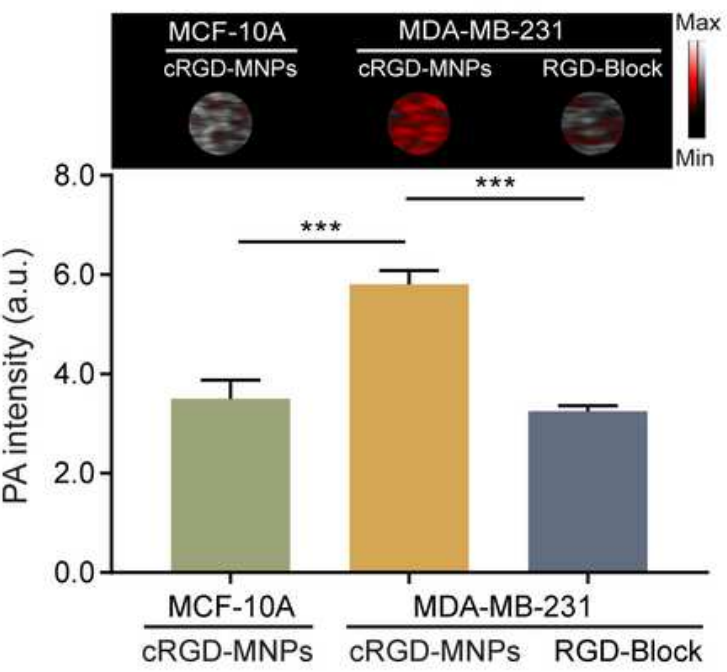

f

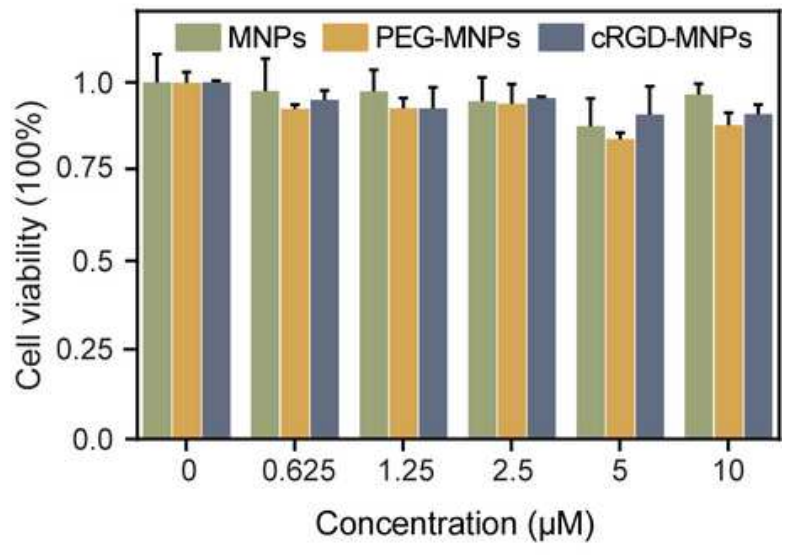

Figure 2

In vitro uptake and cytotoxicity of cRGD-MNPs. a PA images and signal intensities of MDA-MB-231 cells incubated with cRGD-MNPs for various times $(0,1,2,4$ and $8 \mathrm{~h})$. b PA images and signal intensities of 
MDA-MB-231 cells incubated with cRGD-MNPs at various concentrations $(0,0.125,0.25,0.5$ and $1 \mu \mathrm{M})$. C PA images and signal intensities of MDA-MB-231 cells incubated with $0.5 \mu \mathrm{M}$ MNPs, PEG-MNPs or cRGDMNPs for $4 \mathrm{~h}$. PBS was used as a negative control. $d$ PA images and signal intensities of MCF-10A and MDA-MB-231 cells after incubation with cRGD-MNPs for $4 \mathrm{~h}$ with or without free RGD blocking. e CLSM images of MCF-10A and MDA-MB-231 cells incubated with Rho-cRGD-MNPs for $4 \mathrm{~h}$ with or without free RGD blocking. Scale bar: $20 \mu \mathrm{m}$. $\mathrm{f}$ MDA-MB-231 cell viability after incubation with MNPs, PEG-MNPs or CRGD-MNPs at gradient concentrations using standard MTS assay. Data are presented as the mean \pm SD $(n=) 3, * \star * P<0.001$. 

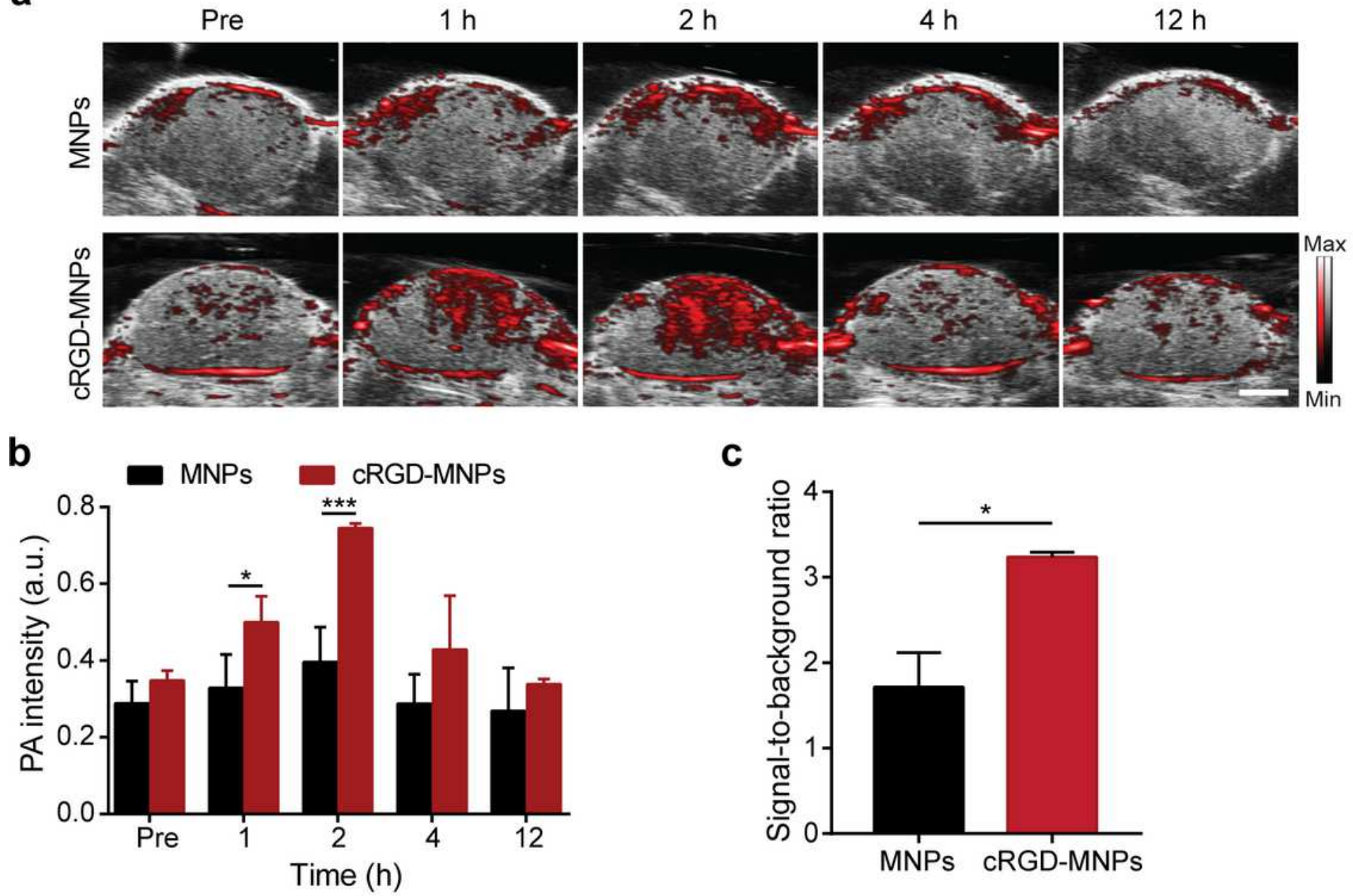

e

d
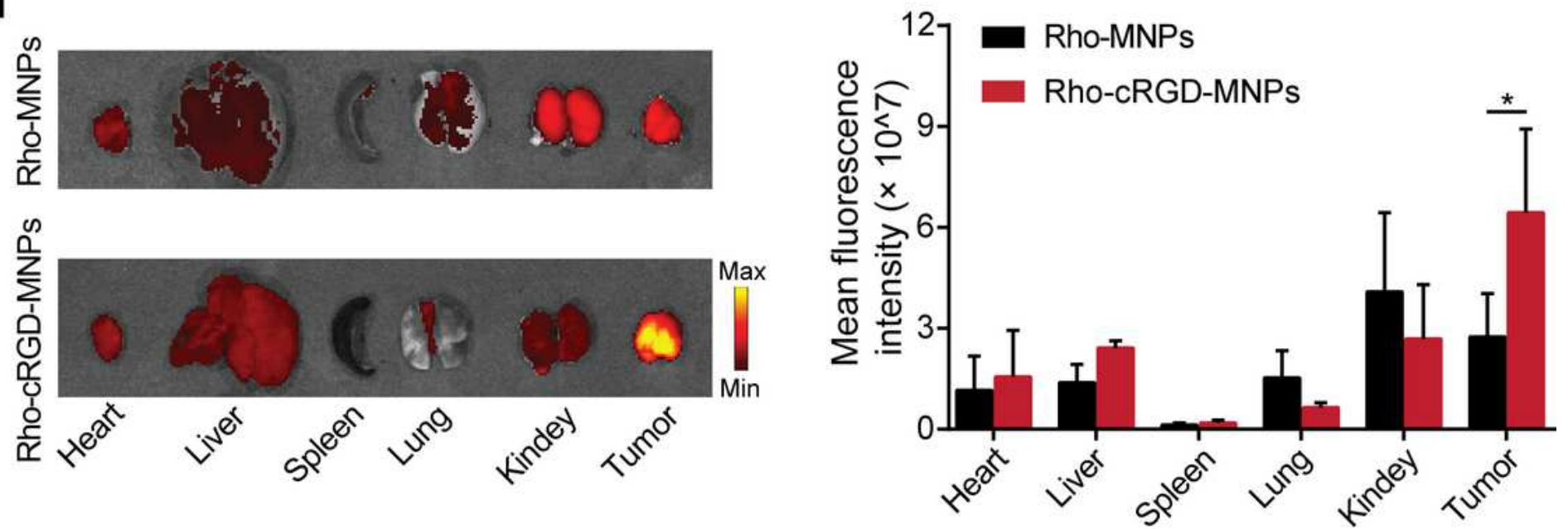

Figure 3

Tumour-targeting and biodistribution of CRGD-MNPs using an MDA-MB-231 xenograft mouse model. a In vivo merged PA and US images of MDA-MB-231 tumour-bearing mice at various times (pre, 1, 2, 4 and 12 h) after intravenous injection of MNPs or CRGD-MNPs $(100 \mu \mathrm{M}, 200 \mu \mathrm{L})$. Scale bar: $2 \mathrm{~mm}$. b Quantitative analysis of PA intensities of tumour sites at the different time points in (a). c Signal-to-background ratio of the tumour region two hours post-injection of MNPs or CRGD-MNPs. $d$ The biodistribution of 
rhodamine-labelled MNPs or CRGD-MNPs in the heart, liver, spleen, lung, kidney and tumour from MDAMB-231 tumour-bearing mice two hours post-injection. e Quantitative analysis of the fluorescence intensities of the tissues in (d). Data are presented as the mean $\pm S D(n=3) ; * \star * P<0.001, * P<0.05$.

a

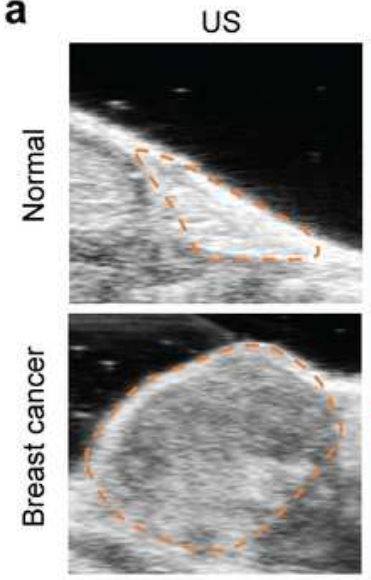

C

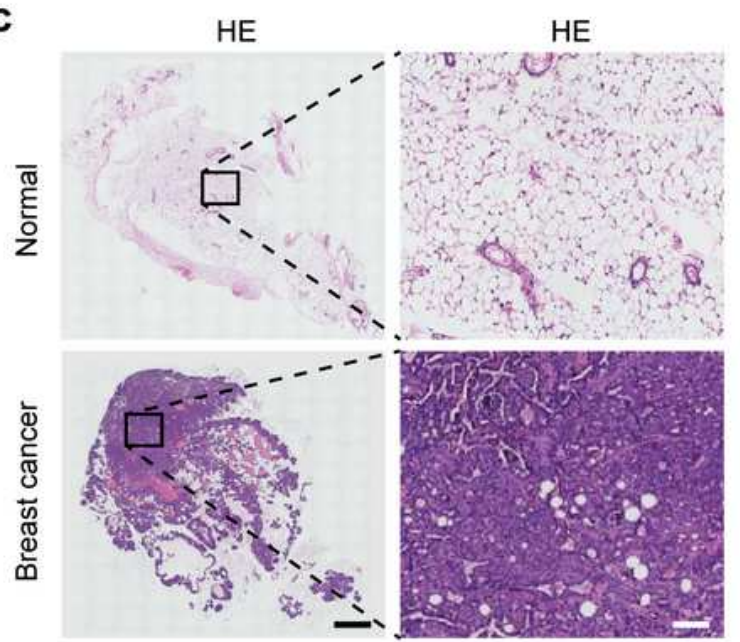

e
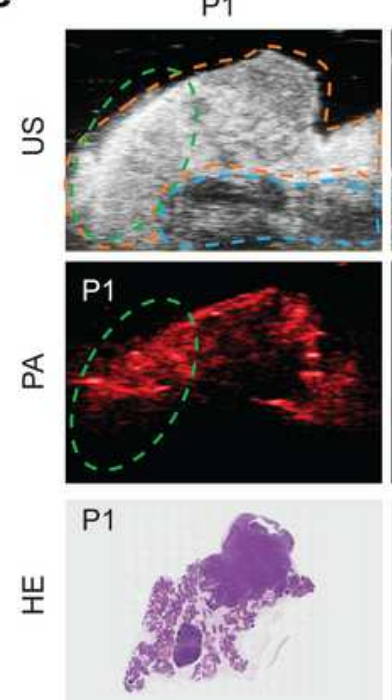

$\mathrm{PA}$
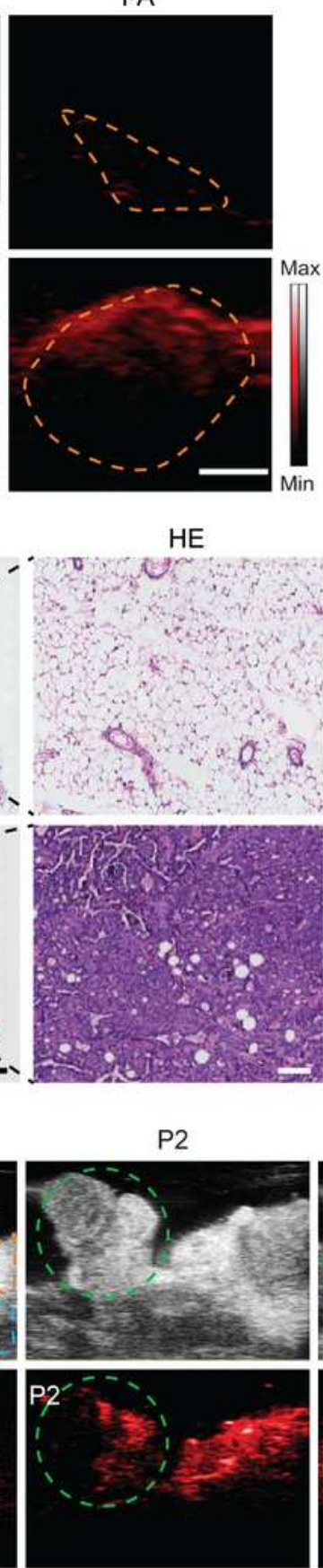

P2

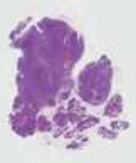

d
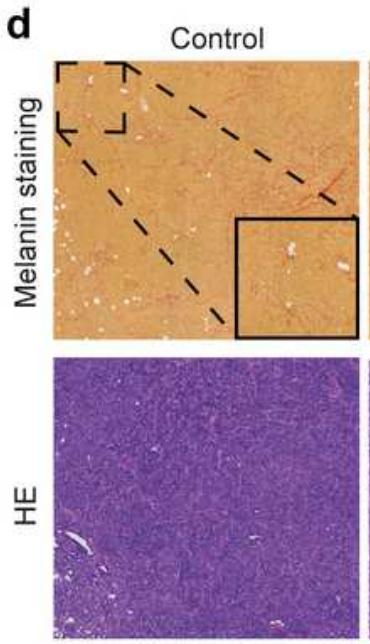

P3

b
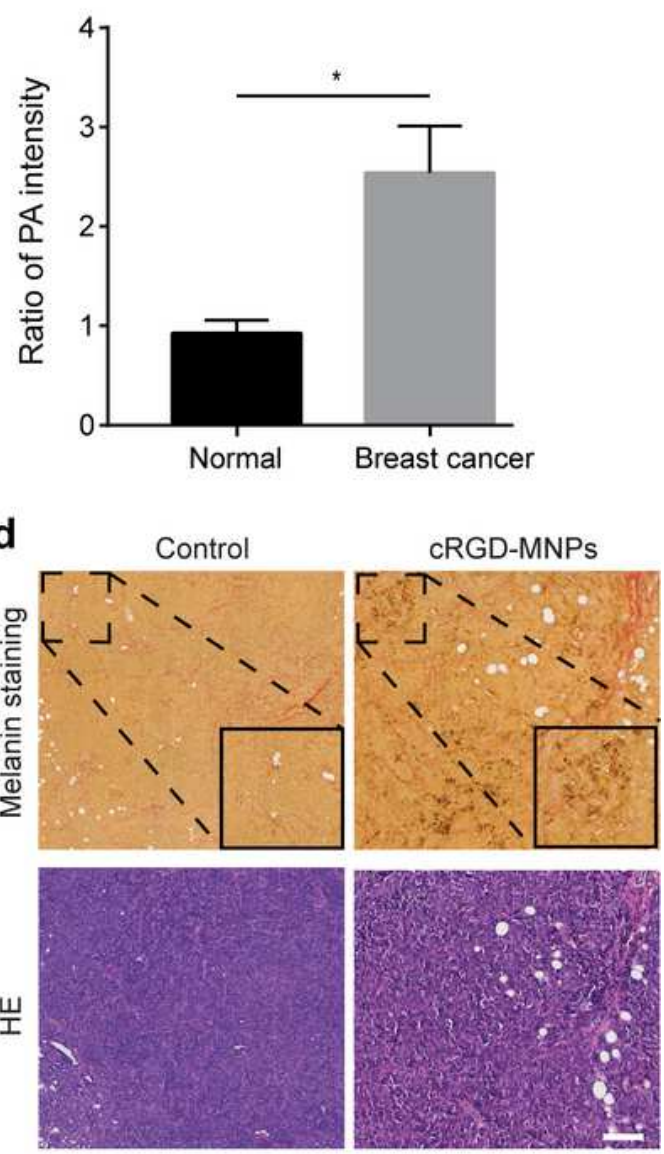

P4

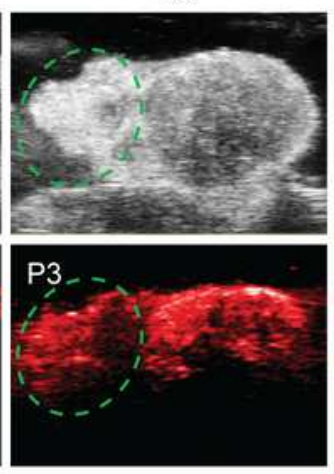

P3

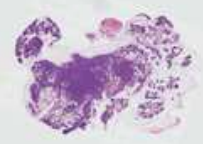

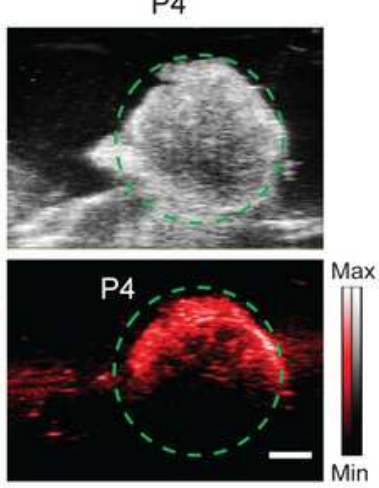

P4

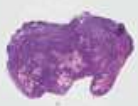

Figure 4

PAl for mammary glands containing spontaneous breast cancer in MMTV-PyVT transgenic mice. a Representative US and PA images of CRGD-MNPs accumulation in mice with normal mammary glands or 
breast cancer. Scale bar: $2 \mathrm{~mm}$. b PA signal intensities of breast tumours and normal mammary glands in transgenic mice two hours after tail-vein injection of cRGD-MNPs compared to $0 \mathrm{~h}$. c Histopathological examination of the tissues from (a). Scale bar: $2 \mathrm{~mm}$. $d$ Fontana-Masson staining of ex vivo tumour tissue. Black particles representing the MNPs were not observed in the non-injected nanoprobe group (left). Black particles representing the CRGD-MNPs were observed in the injection probe group (right). The image in the lower right corner (black square) is an enlarged image of the upper left corner area. Scale bar: $200 \mu \mathrm{m}$. e US (top), PA (middle) and histological (bottom) images of the 4th and 5th pairs of mammary glands in an 8-week-old MMTV-PyVT mouse. The enlarged mammary glands and different regional tissue $(\mathrm{P} 1-4)$ are outlined with dotted orange and green lines, respectively. The dotted blue line outlines the tissue inferior to the mammary gland. Scale bar: $2 \mathrm{~mm}$. Data are presented as the mean \pm SD $(n=3) ; * P<0.05$. 
a

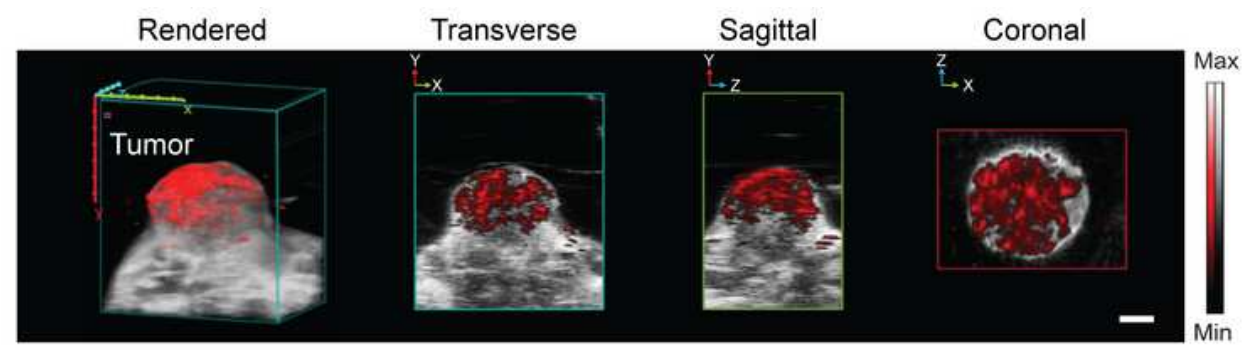

b
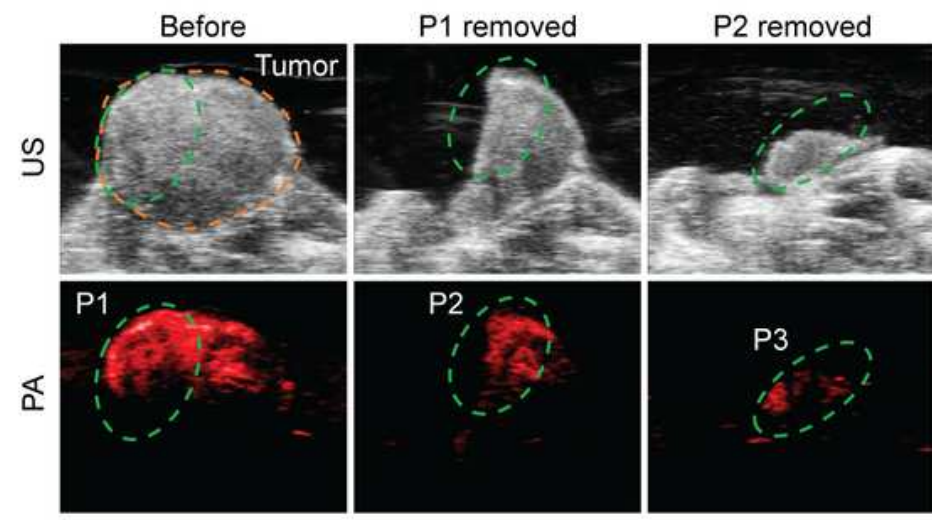

P3 removed
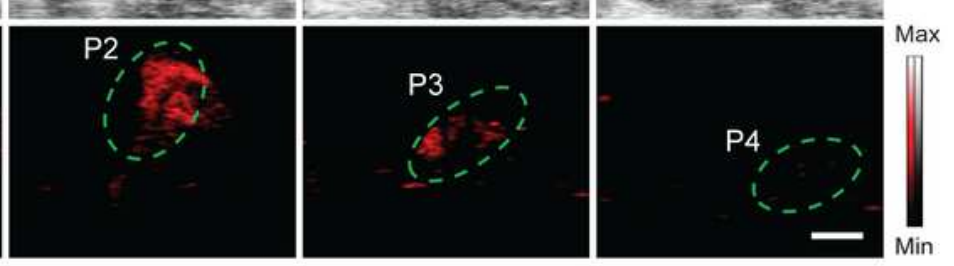

C

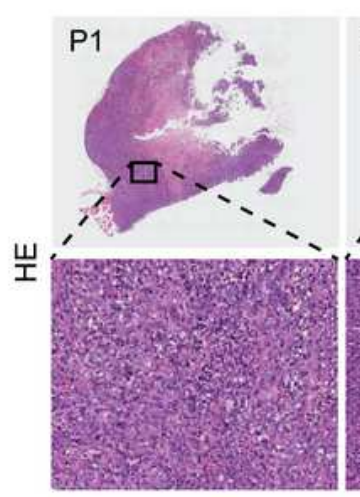

P2

P3

P4

d

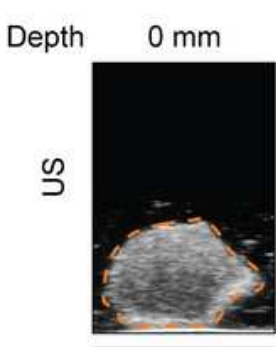

$1 \mathrm{~mm}$
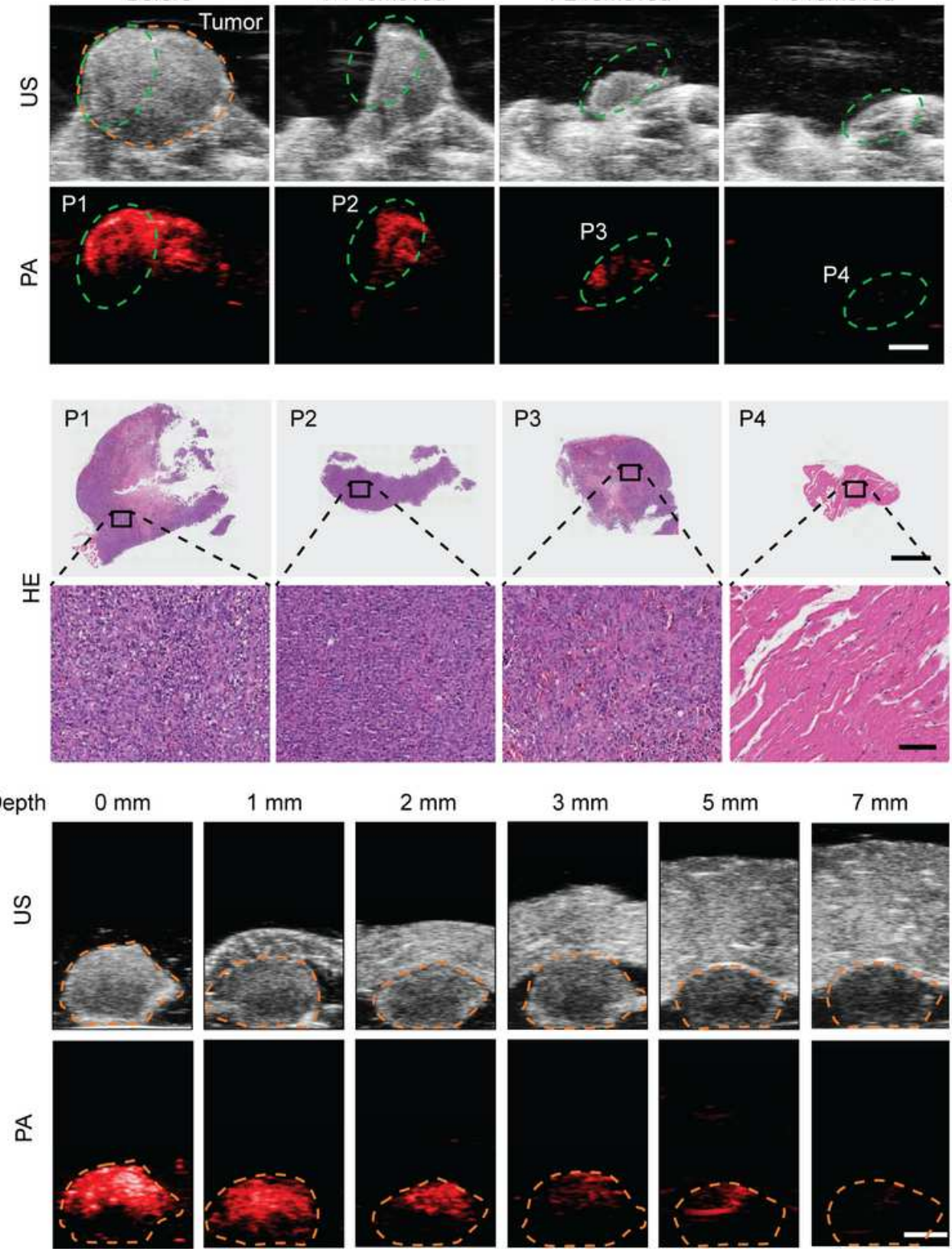

$7 \mathrm{~mm}$
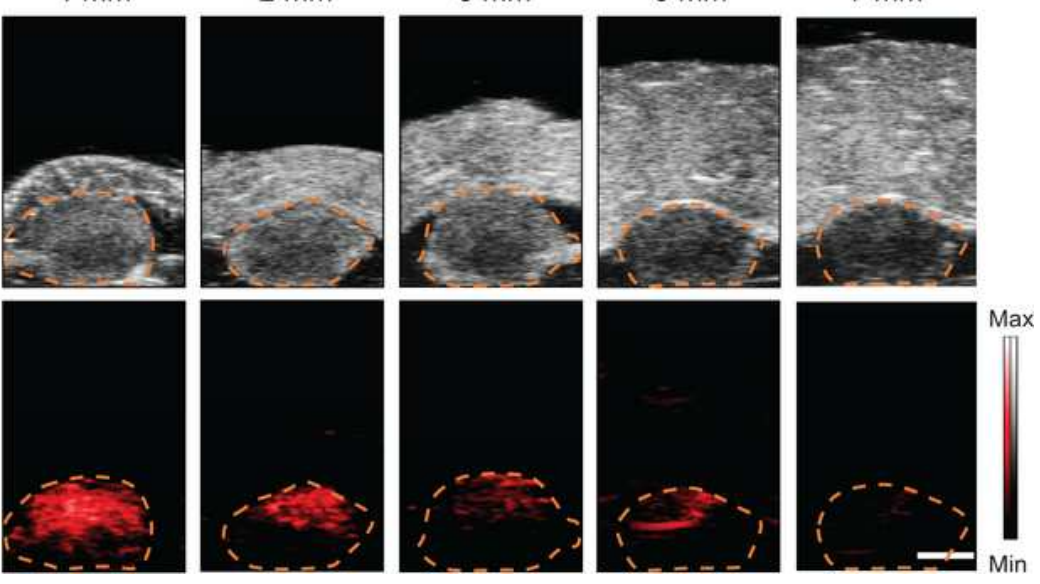

\section{Figure 5}

PAl-guided tumour resection in MDA-MB-231 tumour-bearing mice. a Render, axial, sagittal and coronal images showing the tumour distribution. Scale bar: $2 \mathrm{~mm}$. b Anatomical US (top) and PA (bottom) images showing the tumour region (dotted orange line) in MDA-MB-231 tumour-bearing mice in vivo. The tissue portion to be resected (P1-4) is highlighted with a green dashed circle. Scale bar: 2 mm. c Histological images (top) of resected tissue pieces. Scale bar: $2 \mathrm{~mm}$. Enlarged images of regions marked 
with black boxes are also shown (bottom). Scale bar: $100 \mu \mathrm{m}$. $\mathrm{d}$ PA and US images of tumour tissues covered by different thicknesses of chicken breast under $680 \mathrm{~nm}$ laser excitation in vitro. Scale bar: 2 $\mathrm{mm}$.
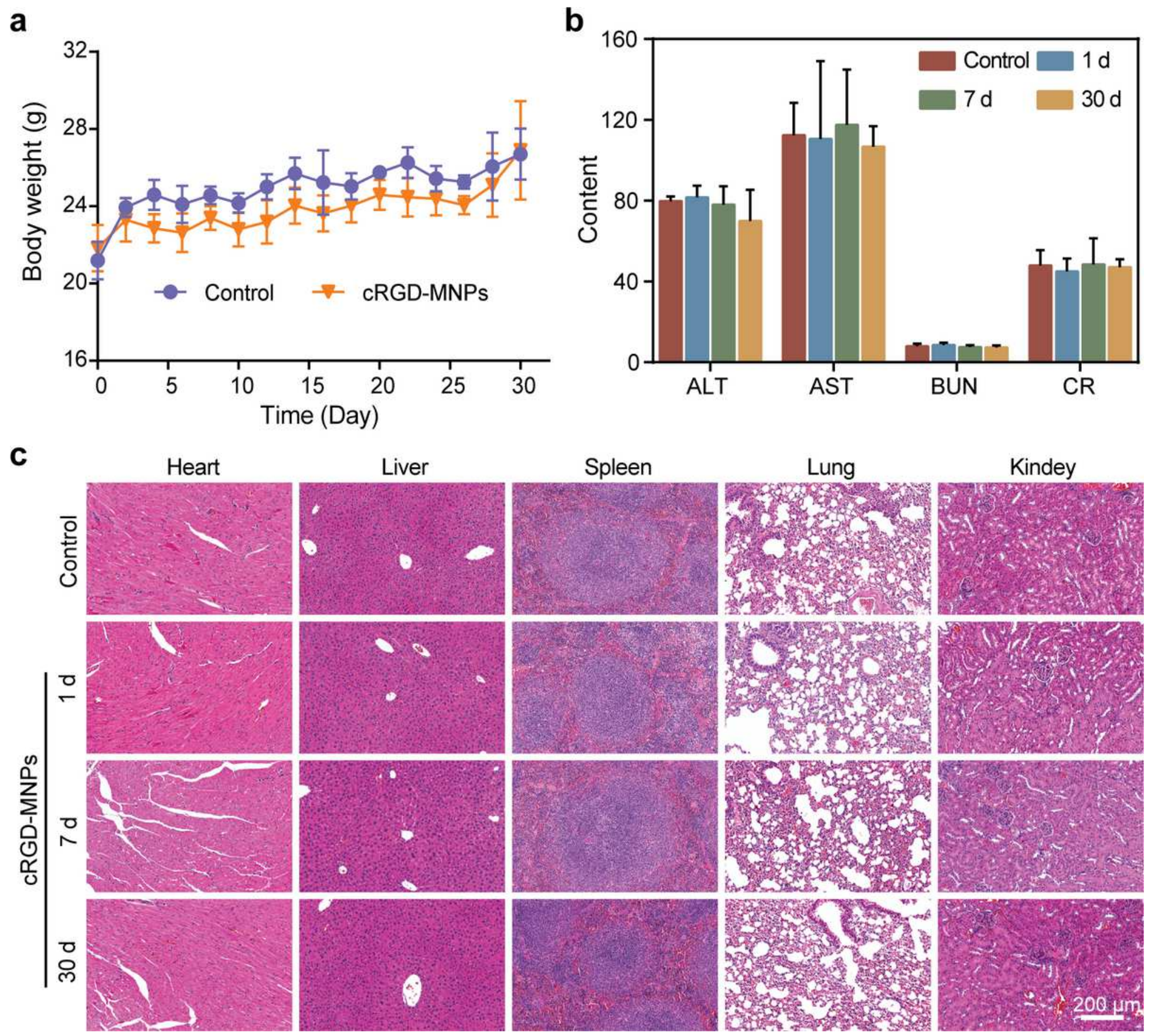

Figure 6

The biosafety of CRGD-MNPs. a Body weight curves for mice treated intravenously with saline (control) or cRGD-MNPs. b Serum biochemistry results (liver function and renal function) for BALB/c mice at various time points (1, 7 and 30 days) after intravenous injection of cRGD-MNPs or saline (controls). The units of ALT and AST are U/L; the units of BUN and CR are $\mathrm{mmol} / \mathrm{L}$ and $\mu \mathrm{mol} / \mathrm{L}$, respectively. $\mathrm{c} \mathrm{H \& E}$ staining of vital organs (heart, liver, spleen, lung and kidney) from each group. Scale bar: $200 \mu \mathrm{m}$. Data are presented as the mean $\pm S D(n=5)$. 


\section{Supplementary Files}

This is a list of supplementary files associated with this preprint. Click to download.

- scheme.png

- Supplementarylnformation.pdf 